\title{
Review Article \\ Optical Measurement Techniques of Recombination Lifetime Based on the Free Carriers Absorption Effect
}

\author{
Martina De Laurentis and Andrea Irace \\ Dipartimento di Ingegneria Elettrica e delle Tecnologie dell'Informazione, Università degli Studi di Napoli "Federico II", \\ Via Claudio, 21, 80125 Napoli, Italy \\ Correspondence should be addressed to Andrea Irace; andrea.irace@unina.it
}

Received 15 November 2013; Revised 21 February 2014; Accepted 3 April 2014; Published 24 June 2014

Academic Editor: George Cirlin

Copyright (C) 2014 M. De Laurentis and A. Irace. This is an open access article distributed under the Creative Commons Attribution License, which permits unrestricted use, distribution, and reproduction in any medium, provided the original work is properly cited.

\begin{abstract}
We review successful measurement techniques for the evaluation of the recombination properties in semiconductor materials based on the optically induced free carrier absorption. All the methodologies presented share the common feature of exploiting a laser beam to excite electron-hole pairs within the volume of the sample under investigation, while the probing methods can vary according to the different methodology analyzed. As recombination properties are of paramount importance in determining the properties of semiconductor devices (i.e, bipolar transistor gain, power devices switching features, and solar cells efficiency), their knowledge allows for better understanding of experimental results and robust TCAD simulator calibration. Being contactless and applicable without any particular preparation of the sample under investigation, they have been considered attractive to monitor these parameters inline or just after production of many different semiconductor devices.
\end{abstract}

\section{Introduction}

The minority carriers recombination lifetime is one of the most important parameters as it both characterizes the semiconductor materials and it strongly influences devices properties. As more than $95 \%$ of all the produced electronic devices are fabricated through CMOS process, nowadays the majority of the studies on recombination lifetime concerns power electronics, diodes, IGBTs-where lifetime killing methodologies are mostly employed-or solar cells, where recombination parameters are directly related to the conversion capability of these devices.

Solar cells, without doubt, occupy an important role in the energy worldwide scenario, so the industry and research interest in their production and characterization are increasing. As said, their efficiency in converting the energy from solar to electrical critically depends on the recombination process by means of two parameters: the bulk recombination lifetime $\tau_{b}$, which accounts for the recombination of electrons and holes inside the silicon crystal, and the surface recombination velocity $S R V$ which is strongly dependent on the interface between the material and its boundaries: the former accounts for material quality, while the latter usually depends on the fabrication process. These parameters are strongly related to the presence of defects within the semiconductor forbidden gap (which directly affects devices performance) and to the surface quality. They also depend on the semiconductor growth technique, on the doping, on the surface condition, and on the free carrier density injected in the material under operating conditions. In the last decades the researchers efforts were addressed to develop contactless and totally compatible methods that allow for monitoring these quantities during the production process without interfering with other fabrication steps. It is thus obvious that methods based on optical or microwave radiations have been preferred.

In this paper we present a review of two important contactless techniques used to determine the semiconductor materials lifetime: the Pump and Probe method (PP) [1] and the Infrared Lifetime Mapping method (ILM), otherwise known as Carriers Density Imaging (CDI) [2, 3]. These techniques are based on the free carriers absorption that occurs in the semiconductor materials. As the name suggests, this is the contribution to the absorption of the free carriers present in the material that involves photons with wavelength belonging to the NIR/middle infrared region, corresponding, 
in terms of energy, to the energy lower than the material band-gap. In fact, the free carriers concentration (and its variation) inside the material determines a variation of the material optical absorption coefficient. This implies that a variation of transmittance can be detected and observed when a sub-band-gap radiation is transmitted by the material. The generation of the excess free carriers can be optically induced by illuminating the sample with a laser pulse, whose photons energy is higher than the sample band gap.

The PP technique belongs to the class of transient methods. These methods are attractive because they give a result directly related to the velocity of the recombination process. In fact, the signal detected is the time dependent signal transmitted by the sample. The PP allows for characterizing materials with very low lifetime too and usually it does not require any calibration procedure. Moreover the actual advantage of the method is the possibility to separate the bulk from surface effect, which means to simultaneously measure the bulk lifetime and the surface recombination velocity.

The ILM/CDI belongs to the steady-state methods class. These methods measure a physical quantity related to the carrier density. It allows for a fast and high spatially resolved measurement of the lifetime in each point of the wafer without performing a scanning of it, since an infrared CCD camera is used to detect the transmitted infrared radiation. Its greater attractive feature is the capability to perform the lifetime mapping in few minutes, which could provide, in calibrated condition, an online monitoring feature.

The paper is organized as follows: in the first two sections we briefly recall the concept of recombination lifetime together with the main recombination mechanisms in semiconductor materials; in the subsequent sections we describe in detail of the selected techniques, having previously recalled the analytical tools necessary to their understanding.

\section{The Concept of Recombination Lifetime}

The term "lifetime" is used in physics in a totally intuitive manner to indicate the temporal interval between the generation and the death of a particle. Nevertheless often the "death" is only a change of the particle state, like in the case of the recombination process in the semiconductors theory. In fact the recombination lifetime refers to the time in which the electron returns from an excited state to the equilibrium state. The excitation can be obtained, for example, by a photon absorption or an electrical injection process in the forward-biased $p-n$ junction. The electron comes back in the equilibrium state, occupying the vacancy left by the excitation. In the semiconductors theory this vacancy is described like a particle, named hole, with the same electron charge but different mass $[4,5]$. In this way the electron decay to the equilibrium state is described as its recombination with the hole. This recombination process destroys the charge constituted by the pair and the recombination lifetime then refers to the electron-hole pair: what ceases to exist is this charged pair and it ceases to exist because the electron of the pair recombines with the hole of the pair. In other words, the recombination process is the destruction (or decay) of the excess carriers (the electron-hole pairs) generated in the semiconductors as consequence of an excitation. The temporal interval during which the excess carriers decay is defined as recombination lifetime, $\tau_{\text {rec }}$.

Mathematically, in a first order approximation, $\tau_{\text {rec }}$ is defined as the ratio

$$
\tau_{\text {rec }}=\frac{\Delta n}{\mathscr{R}}
$$

between the excess carriers density $\Delta n\left[\mathrm{~cm}^{-3}\right]$ and the recombination rate $\mathscr{R}=-d(\Delta n) / d t\left[\mathrm{~s}^{-1}\right]$ of the excess carriers itself.

From (1), named $\Delta n(0)$, the excess carriers concentration at the instant $t=0$ when the excitation source is turned off, it is derived that $\tau$ characterizes the excess carriers decay:

$$
\Delta n(t)=\Delta n(0) \exp \left(-\frac{1}{\tau_{\text {rec }}}\right)
$$

In steady-state conditions the excitation source is at all times turned on, constant or with assigned time dependence, and an equilibrium condition between the generation rate and recombination rate is reached, $\mathscr{G}=\mathscr{R}$, so that it is possible to define the recombination lifetime as

$$
\tau_{\text {rec }}=\frac{\Delta n}{\mathscr{G}}
$$

In general it must be clearly remarked that the recombination phenomenon can be comprehensively treated only by means of quantum mechanical calculations. Since it involves transitions between energetic states, it is implicit that the recombination rate depends on occupation probability of the energy levels, and, thus, on the energy as well as on the temperature, on the electrons, and holes concentration, and it is in general a nonlinear function of $\Delta n$. Consequently the recombination lifetime depends on them and thus it must be considered that in general it is $\tau_{\text {rec }}=\tau_{\text {rec }}(E, T, n, p, \Delta n, \Delta p)$ (where $n$ and $p$ are the concentration of the electrons and holes, resp.); that means, among other things, that $\tau_{\text {rec }}$ is a function of the injection level $\Delta n / n(\Delta p / p)$.

2.1. Recombination Process. The total recombination rate is a superposition of several recombination mechanisms. As summarized in (4), among these the main are the ShockleyRead-Hall, the radiative, and the Auger recombination (the first one linearly dependent on the excess carrier generation only at lower injection level, the second one and the third, resp., proportional to the square and to the cube of the excess free carriers concentration):

$$
\mathscr{R}=\mathscr{R}_{\mathrm{SRH}}+\mathscr{R}_{\mathrm{Rad}}+\mathscr{R}_{\mathrm{Aug}}
$$

The sketch in Figure 1 gives an intuitive $E-k$ picture of these processes, while in the following we give a short description of them. We invite the readers to refer to $[6,7]$ and especially to [8] for all the analytical and theoretical details. 




(a)



(b)

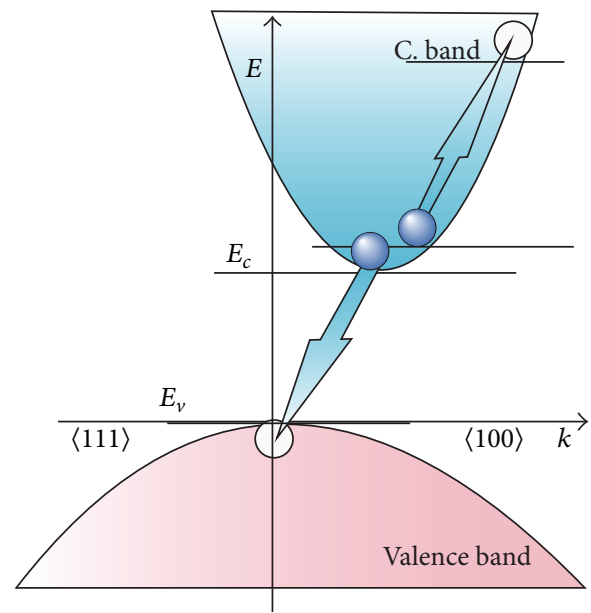

(c)

Figure 1: Main Recombination Mechanisms in semiconductors: (a) Trap-assisted recombination. (b) Direct Recombination. (c) Auger Recombination.

2.1.1. Shockley-Read-Hall Recombination. Shockley, Read, and Hall in 1952 were the first to note that, in the indirect band-gap semiconductors, like silicon and germanium, the recombination lifetime is sensitive to the material property and that the recombination rate varies linearly with the carriers concentration on a wide range of concentrations and temperatures $[9,10]$. Since this cannot be explained with a two-bodies collision mechanism, adopted to explain the interaction between the electrons and which implies a quadratic dependence, they supposed and demonstrated that the process takes place by means of the material impurities that introduce some intermediate energy levels in the forbidden gap. These levels act as recombination centers (or traps, from which the process is otherwise depicted as trap assisted recombination) and an intermediate step is introduced in the recombination process.

Therefore the electron in a first step falls down to the intermediate levels; then it falls down to the valence band in the same way. Nevertheless, since the process is observed in the materials called indirect semiconductors, because of noncorrespondence in the momentum-space $(k$-space $)[4,5]$ between the maximum of the valence band and the minimum of the conduction band, an electron in the conduction band cannot simply decay to the valence band releasing its energy, but it must also change its momentum. This can happen with the assistance of the crystal lattice: the electron gives the lattice its energy in the form of heat, and, simultaneously, this interaction allows it to change its momentum $k$, producing lattice vibration. In the quantum model this is described as the interaction of the electron with the lattice vibrational quanta, the phonons. This fictitious particles are defined with an own momentum [4], so that when the electron interacts with them, it can change its momentum to preserve the total momentum of the whole particle.

The second step can be equivalently described as an hole that ascends to the intermediate level (equivalently described as an "hole capture" of the trap), recombining with an electron. Really, depending on the nature of the trapping 
process, one of the two steps can be a radiative process, so that the electron losses its energy emitting a photon. This process does not imply a momentum change.

It must be noted that the trap assisted recombination is a very complex phenomenon that can be properly described by means of the quantum mechanics and, we remark, it can be assumed linearly dependent on the excess carriers concentration only on a limited, even if wide, range of concentrations and temperatures. The readers can find a complete and exhaustive treatment in $[8,11]$.

2.1.2. Radiative Recombination. In direct band-gap semiconductors, where the minimum of the conduction band coincides with the maximum of the valence band, the electron band to band transition can happen without involving the crystal lattice, but only with the emission or absorption of a photon, depending on the kind of process (if it is an absorption or a decay). This is possible because the change of momentum is not required and thus only the energy conservation must be preserved.

Since the process simultaneously involves both the charges, electrons and holes, the probability that it occurs is proportional to the square of the population of the two involved levels and, likewise, the recombination rate too.

2.1.3. Auger Recombination. In 1955 Pincherle published a brief letter on the Proceeding of Physical Society [12] in which the inverse proportionality of the minority carriers density lifetime to the square of the majority carriers density, experimentally observed by Moss in impure lead sulfide (PbS) [13] in 1953 and by Hornbeck and Haynes in silicon (Si) in 1955 [14], was justified and predicted by means of the Auger effect [15]. In the subsequent years between 1956 and 1958 Landsberg et al. [16-18] developed the first theory on the Auger recombination.

The Auger recombination in semiconductors involves three carriers: when an electron recombines with a hole, the energy and the momentum variation are transferred to a third carrier, either an electron or a hole. The conduction band Auger recombination (case " $\mathrm{c}$ " of the figure), which involves two electrons in the conduction band and a hole in the valence band, is sketched in Figure 1. In a similar way, the valence band Auger recombination involves one electron in the conduction band and two holes in valence band. The Auger recombination can involve trap levels too $[8,19-21]$. The process is strongly dependent on the carriers injection level and it becomes dominant at high carriers concentration.

From this short summary on the recombination mechanism and recalling (1) and (4), it is possible to derive that that the recombination lifetime can be expressed as

$$
\frac{1}{\tau_{\text {rec }}}=\frac{1}{\tau_{\text {SRH }}}+\frac{1}{\tau_{\text {rad }}}+\frac{1}{\tau_{\text {Auger }}} .
$$

In indirect band-gap semiconductors, like $\mathrm{Si}$, the $\mathrm{SRH}$ is dominant at moderate carrier densities (up to $10^{18} \mathrm{~cm}^{-3}$ ), while the Auger recombination dominates under high doping and high injection condition (see, e.g., [22]). This implies that $\tau_{\text {rad }}$ is enough higher than $\tau_{\mathrm{SRH}}$ and $\tau_{\text {Auger }}$ and thus in (5) the second term is usually negligible. Radiative recombination dominates in direct band-gap materials, like GaAs and InP.

2.2. Surface Recombination Lifetime and Surface Recombination Velocity. The materials' samples and the devices (or the active region of them) are space limited by their surfaces, which constitute an interface between two different media, like the silicon-air surface or the silicon-oxide surface. Likewise to the impurities and to the defects, these surfaceswhich are discontinuities of the periodical crystal structureintroduce allowed energy states within the forbidden band. These states affect the recombination process in a way that is very similar to the Shockley-Read-Hall model for the bulk recombination. This kind of recombination is known as surface recombination and it is characterized by an own recombination lifetime $\tau_{S}$.

Usually all the experimental techniques determine a parameter that is a combination of the bulk contribute $\tau_{b}$ and of the surface one, called effective lifetime:

$$
\frac{1}{\tau_{\mathrm{eff}}}=\frac{1}{\tau_{b}}+\frac{1}{\tau_{S}} .
$$

Nevertheless to characterize the recombination at the surface, rather than $\tau_{S}$ another parameter is usually used, the so-called surface recombination velocity or SRV. Anyway, without entering in detail, for which we refer to [8, 23-27], to define SRV we have to consider that, since the carriers that recombine at the surfaces of the sample can be described as a current-like flow outside the latest, it is much easier to model the presence of the recombination centers on the surface by means of appropriate boundary conditions that bind the values of the carriers concentration and its gradient at the boundaries. This leads to the following SRV definition:

$$
\mathrm{SRV}=D\left[\frac{\partial n / \partial x}{n(t)}\right]_{\text {boundary }},
$$

if $x$ is the space coordinate and a $1-D$ problem is under consideration, being $D$ the carriers ambipolar or monopolar diffusion coefficient, as determined by the carriers injection level.

Even if the majority of the experimental cases are well explained with a constant SRV, a more complicated theory can be taken into account when a nonnegligible bandbending is present at the surface [26].

\section{Free Carriers Absorption}

In semiconductor materials the injected or generated free carriers contribute to the absorption process by means of intraband transitions. It is known that three absorption mechanisms happen in these kinds of materials. We briefly remember them.

(i) Intrinsic or Band-gap Absorption occurs in the intrinsic semiconductors when photons with energy $E_{\mathrm{ph}} \geq$ $E_{g}$ ( $E_{g}$ is the material band gap) irradiate the material. This absorption determines a transition from the 
valence band to the conduction one. The conventional visible and NIR photodetectors are based on this effect.

(ii) Impurity Level to Band Absorption occurs in extrinsic materials, like in the extrinsic infrared photodetectors, when photons with energy $E_{i} \leq E_{\mathrm{ph}} \leq E_{g}$ impinges on the material. The photons absorption determines transition from the impurity intraband energy level to the band, as the name suggests. This phenomenon is used, for example, to tune the wavelength response of the IR detectors, by choosing impurities with different energy level belonging to the material band-gap.

(iii) Free Carriers Absorption. The free carriers present in the semiconductor materials contribute to the absorption process, absorbing photons with energy $E_{\mathrm{ph}} \leq E_{\mathrm{g}}$ (that corresponds to the middle or far infrared region) and determining intraband transition. This phenomenon can degrade the detectors response, since the absorption increases as power function of the wavelength. Nevertheless, it is useful to measure the carriers concentration, with this effect being proportional to them.

The FCA has been widely studied in the middle of the 20th century, by means of both classical Drude model and quantum one (see, e.g., [28-39]).

The absorption of a photon with energy smaller than the energy band-gap of the semiconductor material allows the free carrier to have a transition from a lower energetic state of the band to a higher energetic state of the same band. Nevertheless, since the two involved states belong to the same band, the transition can occur only with a momentum change. This change is possible by means of interactions of the free carriers with the material lattice (lattice vibration described by means of phonons) or by means of scattering from ionized impurities. The classical theory, based on the Drude model, describes the free carriers in the solid like a gas of particles with a density $n$, subjected to collisions with the relatively fixed ions of the lattice in which it is moving. It leads to a dependence of the free carriers absorption coefficient on the carriers concentration and on the square of the wavelength of the absorbed photons:

$$
\alpha_{\mathrm{fc}}=\frac{n e^{2} \lambda^{2}}{m_{e} \epsilon_{0} \eta 4 \pi c^{3}} \frac{1}{\tau},
$$

where $e$ is the electron charge, $m_{e}$ is the carrier effective mass, $\epsilon_{0}$ is the vacuum permittivity, $\eta$ is the material refractive index, $c$ is the light speed, $\lambda$ is the wavelength of the absorbed photons, and $\tau$ is the relaxation time, which represent the average time between two subsequent collisions, or, in other words, the free motion time of the gas particle before another collision with the lattice ions happens.

In the previous expression the relaxation constant is assumed independent of the energy, but really it is a complicated function of the energy and contains the details of the collision mechanism. These details have been investigated by means of the quantum theory, studying the interaction mechanisms between the carriers and the semiconductors lattice which are described as interaction between the carriers and the vibrational modes of the lattice. Moreover the quantum theory of the free carriers absorption justifies the often observed deviation from the experimentally observed $\lambda^{2}$ dependence in some materials and compounds. In general, without entering the details of the calculation and of the phenomenon description, we can synthesize-like in [40]that the main scattering process involving the free carriers in the semiconductors materials contributes with a resultant dependence of the absorption coefficient on the wavelength. The latest can thus be expressed by a weighted sum of few terms, like

$$
\alpha_{s}=A_{s} \lambda^{p_{s}}
$$

where the label $s$ refers to a particular kind of scattering process and each $A_{s}$ is constants dependent on the carriers concentration and on the temperature as well on other parameters, like the carriers effective mass. The impurities concentration in the material determines the dominant mode of scattering and the relative value of the exponent $p$ of the wavelength. Moreover the value should increase with the doping. Typical values are between 1.5 and 3.5. Some examples are given in literature by studying the different kind of scattering and considering different materials and compounds. For example, Fan and Becker [29] reported a value $p=1.5$ for the scattering by the acoustic phonons in silicon and germanium, while in [35] the quantum mechanical calculation of the free carriers absorption, considering the scattering by the optical phonons in the III-V compounds, is presented and experimentally verified, finding a value $p=$ 2.5. Furthermore [33] shows that the scattering by ionized impurities can lead to $p=3$ or $p=3.5$.

The details of the calculations are reported in the cited papers and also in many books, like in [41], where it is remembered that a tabulation of the expression for $\alpha_{\mathrm{fc}}$ for the several scattering mechanisms is given in [42].

An experimental determination of the free carrier absorption coefficient for the silicon, at fixed temperature of $350 \mathrm{~K}$, was given by Schroder et al. in 1978 [39]. In accordance with the theoretical classical model, the following dependence on the free electrons and holes densities $n$ and $p$ in the $n$-type and $p$-type material, respectively, was found at

$$
\begin{gathered}
\alpha_{n} \simeq 1 \times 10^{-18} \lambda^{2} n, \\
\alpha_{p} \simeq 2.7 \times 10^{-18} \lambda^{2} p,
\end{gathered}
$$

where the wavelength $\lambda$ is measured in $\mathrm{cm}$.

The experimental dependence on the wavelength at different doping levels in the $n$-type silicon and $p$-type at $300 \mathrm{~K}$ can be found in [34] and [37], respectively, while the free carrier absorption versus wavelength for high purity $\mathrm{Si}$ at different temperatures was reported in [38]. 


\section{Free Carriers Absorption Methods to Measure and Map the Recombination Lifetime in Semiconductors Materials}

The intrinsic bond between the free carriers concentration and the optical absorption change, as previously seen, had offered the possibility to directly monitor the former, measuring the latter. Moreover the great appeal in the use of this effect was and is the possibility to measure the quantities related to the carriers density (like the recombination lifetime) and to measure them in an absolutely not invasive and contactless way, so that the sample integrity is totally preserved.

On this principle many of techniques that allow for measuring the recombination lifetime in semiconductors materials are based. In practice these techniques operate with a common principle that makes use of a sub-band-gap radiation to monitor the absorption variation in the sample due to the excitation of an ultra-band-gap radiation. So that the sub-band-gap radiation constitutes the sensitive element in the measurement, that is, the probe, while, adopting the habitual nomenclature used in optics to name an exciting source, the ultra-band-gap radiation constitutes the pump. In this way all the techniques based on FCA can be classified as Pump and Probe. We can refer to them as the FCA PP techniques. One of the first applications can be seen in two works published in 1966 by Chiarotti and Grassano [43, 44]. In 1970 Gauster and Bushnell [45] used the technique to derive the silicon absorption cross section and suggested that the method can be useful to investigate a variety of processes such as the multiphonon absorption, the recombination of the carriers, and the measurement of the carriers lifetime. Interesting in their work is the use of a pulsed pump beam.

FCA PP techniques can be either steady-state methods or dynamic (we will refer to the former with Steady-State PP or easily SS PP methods, while to the latter with Transient PP, or TPP). In the SS PP case the measurement is performed with the pump on or with a modulated pump (so that the lock-in detection can be used to increase the signal to noise ratio) and what is directly observed in the measurement is the distribution of the free carriers when the steady state has been established. The recombination lifetime is defined by the relation (3), where the generation rate $\mathscr{G}$ is proportional to the pump intensity. This requires a calibration performed comparing the distribution without the pump excitation and with the pump excitation, so that the variation of the carriers density can be evaluated and then the related quantities, like the actual recombination lifetime. ILM and the Modulated Free Carriers Absorption [46-50] belong to the Steady-State PP methods. In the case of the TPP, to infer the value of the recombination lifetime in the sample, a pulsed pump is used. In fact, by recording the signal of the detected probe beam just in the transient time after the turn off of the pumb beam, the sample recombination lifetime can be estimated. We will describe it in detail in the following sections together with the ILM. They are, in our opinion and in accordance with our experience, the most consolidate and efficient, being also perfectly complementary regarding their range of application.
We will see that the major advantage of the TPP technique is the possibility to discriminate the bulk contribution from the surface contribution with only one measurement, whereas, on the other hand, as the name said, the ILM can give in one measurement the mapping of the actual lifetime mapping of the whole sample (the use of the adjective "actual" will be clear in the Section 7). Both techniques can operate in low injection regime.

\section{Transient Pump and Probe Technique to Measure the Recombination Lifetime in Semiconductors}

The experimental Transient Pump and Probe scheme to measure the recombination lifetime was well settled by Ling et al. in 1987 [1], but only with subsequent works [51-54], based on the analytical analysis of the interaction of a pulse laser beam with a semiconductor wafer performed by Luke and Cheng [55], the potentialities of the technique were clear. These consist in the capability to discriminate between the surface contribution to the recombination and the bulk one with only one measurement, or, in other words, to measure simultaneously the bulk recombination lifetime and the surface recombination velocity. Moreover, performing more than one measurement with little changes in some parameters, other characteristic electrical parameters of the materials can be retrieved.

Two schemes of Pump and Probe setup have been proposed, depending on the angle between the pump and probe beams: the transverse configuration and the parallel configuration. The parallel configuration is more suitable for wafer lifetime mapping, obtained by scanning the sample along the plane orthogonal to the beams incidence plane $[56,57]$. In this case the diffusion length of the free carriers in the sample, $L=\sqrt{D \tau}$, limits the resolution of the system. In fact, in order to have a right interpretation of the signal the spot size of the probe beam must be more than or equal to the expected diffusion length. Otherwise, if the spot size is smaller, the observed signal is more influenced by the diffusion than by the recombination. Nevertheless if the probe beam is highly focused on the sample, the desired experimental condition can be easily obtained.

In the perpendicular configuration the sensitivity of the measurement is substantially increased by making a long path of interaction between pump and probe beam. This path is, in principle, only limited by the spot size of the pump beam [58]. Since there are no stringent requirements concerning the state of wafer surface, the use of a transverse probe is also attractive, if the conditions of the sample do not allow for an efficient coupling of the probe radiation with the crystal, as in the case of a sandblasted wafer surface, or if special surface treatments are present and need to be characterized or cannot be removed for any reason. In both cases, as previously said, the pump photons energy is higher than the wafer band-gap $\left(\lambda_{p}>\lambda_{\text {gap }}\right)$, but its intensity is not so high to determine a high injection level (Figure 2). In this condition all parameters involved in the recombination process can be assumed to be constant during the time decay. 


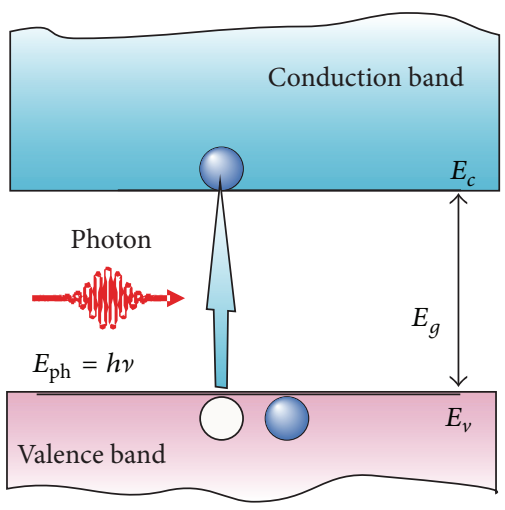

(a) $E_{\mathrm{ph}}>E_{g}$

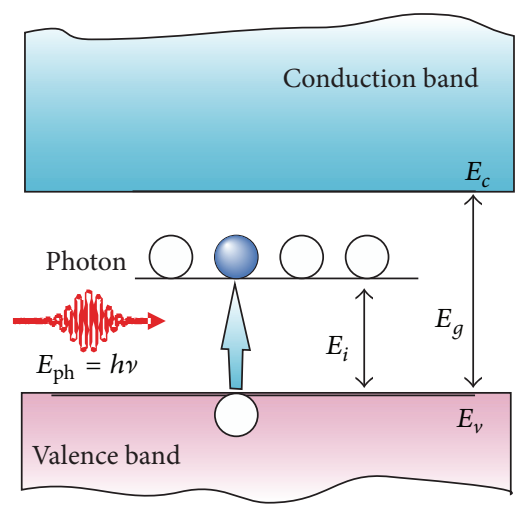

(b) $E_{i}<E_{\mathrm{ph}}<E_{g}$

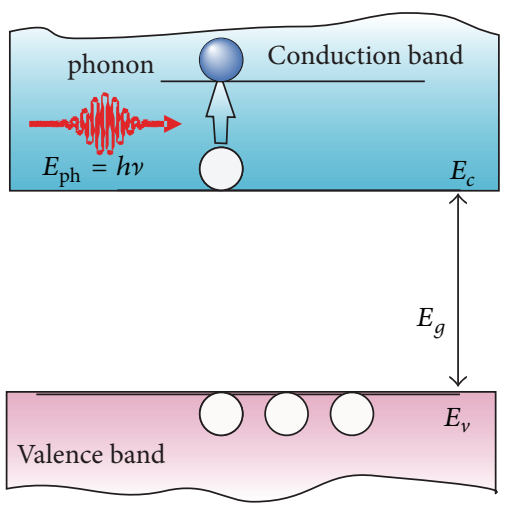

(c) $E_{\mathrm{ph}}<E_{g}$

FIGURE 2: (a) Intrinsic or band-to-band absorption occurs when the energy of the absorbed photon is greater than the material band-gap. (b) Extrinsic or impurity level to band absorption occurs in extrinsic materials when the photon energy is greater than the impurities energy but smaller than the energy band-gap. In the figure the process is sketched in the case of a p-type semiconductors. (c) Intraband or free carriers absorption can occur when the photon energy is smaller than the energy band-gap.

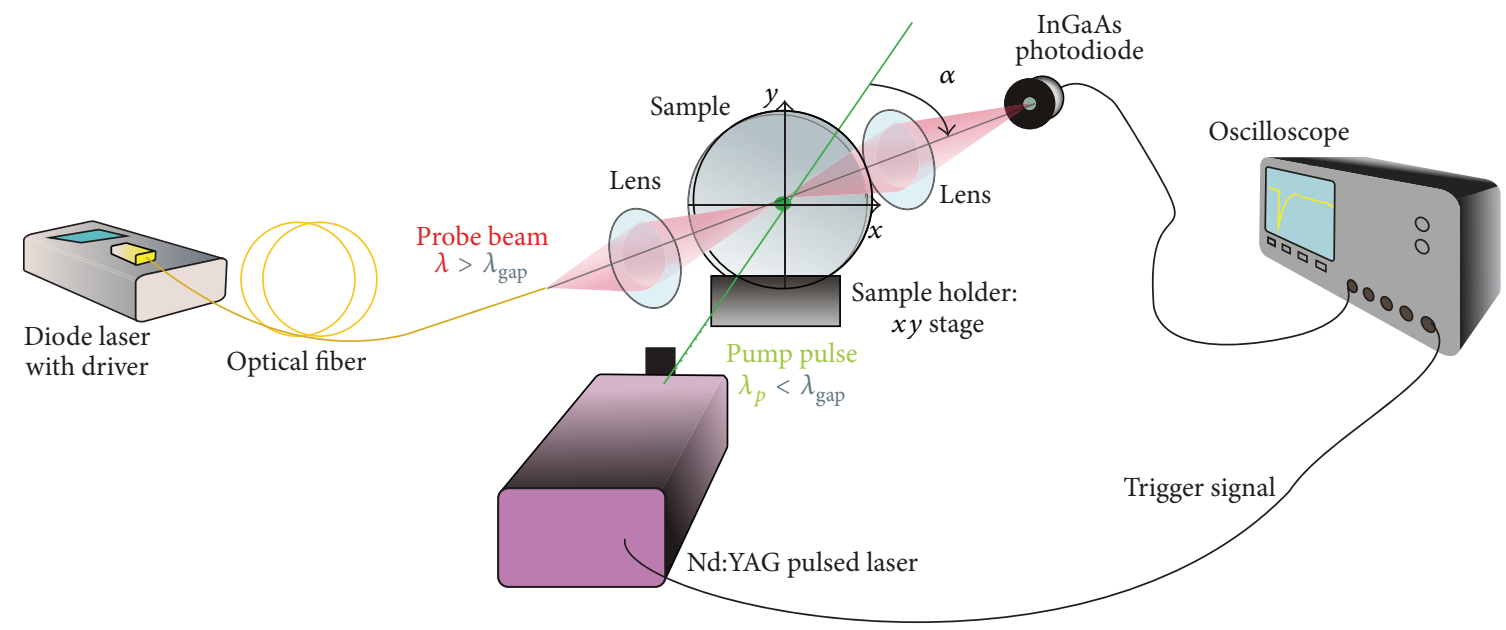

FIgURE 3: Pump and Probe setup in the parallel configuration. The pump pulsed beam and the probe beam impinge on the sample surface. The probe beam is focused on the sample surface, attenuated by the sample, and then detected by the InGaAs photodiode. The diffusion length determines the lateral resolution for the lifetime mapping, so that the probe spot size must be chosen to be more than or, at least, equal to it [56].

A sketch of the parallel and transverse configuration of TPP setup is shown in Figures 3 and 4, respectively. The continuous probe laser beam (usually a laser diode beam $@ 1550 \mathrm{~nm}$ ) is detected by a photodiode after the propagation inside the sample (parallel to sample surface in the case of transverse configuration and orthogonal to it in the other case). Triggered on the pulse pump, the decay curve acquired by the InGaAs detector,

$$
I(t)=I_{0} e^{-\alpha_{0}(\lambda) L}\left(1-e^{-\alpha_{\mathrm{fc}}(\lambda, t) L}\right),
$$

is composed of two contributions: the initial material absorption coefficient, $\alpha_{0}(\lambda)$, and the time dependent free carriers absorption coefficients at the probe wavelength, $\alpha_{\mathrm{fc}}(\lambda, t)$.

In Figure 5 a typical signal of the transmitted probe radiation in which the temporal dependence of the absorption can be observed once the pump is turned off is shown.
To clarify how the Transient PP method works, it is useful to recall the analysis of the interaction of a laser pulse with a semiconductors sample. We report in the following the analysis as presented in [52], where the study of Luke and Cheng [55] is extended to the case of different SRV on the two surfaces of the wafer.

5.1. Modeling of the Analysis of the Interaction of a Laser Pulse with a Silicon Wafer. Let us consider a semiconductor wafer with a thickness $d$, and a laser pulse impinging on its surface 1, as shown in Figure 6. Let $D$ be the minority carriers diffusion coefficient in the material, $S_{1}$ and $S_{2}$ the surface recombination velocity on the surfaces 1 and 2 , respectively, and let us consider the same coordinate system as reported in the cited figure. 


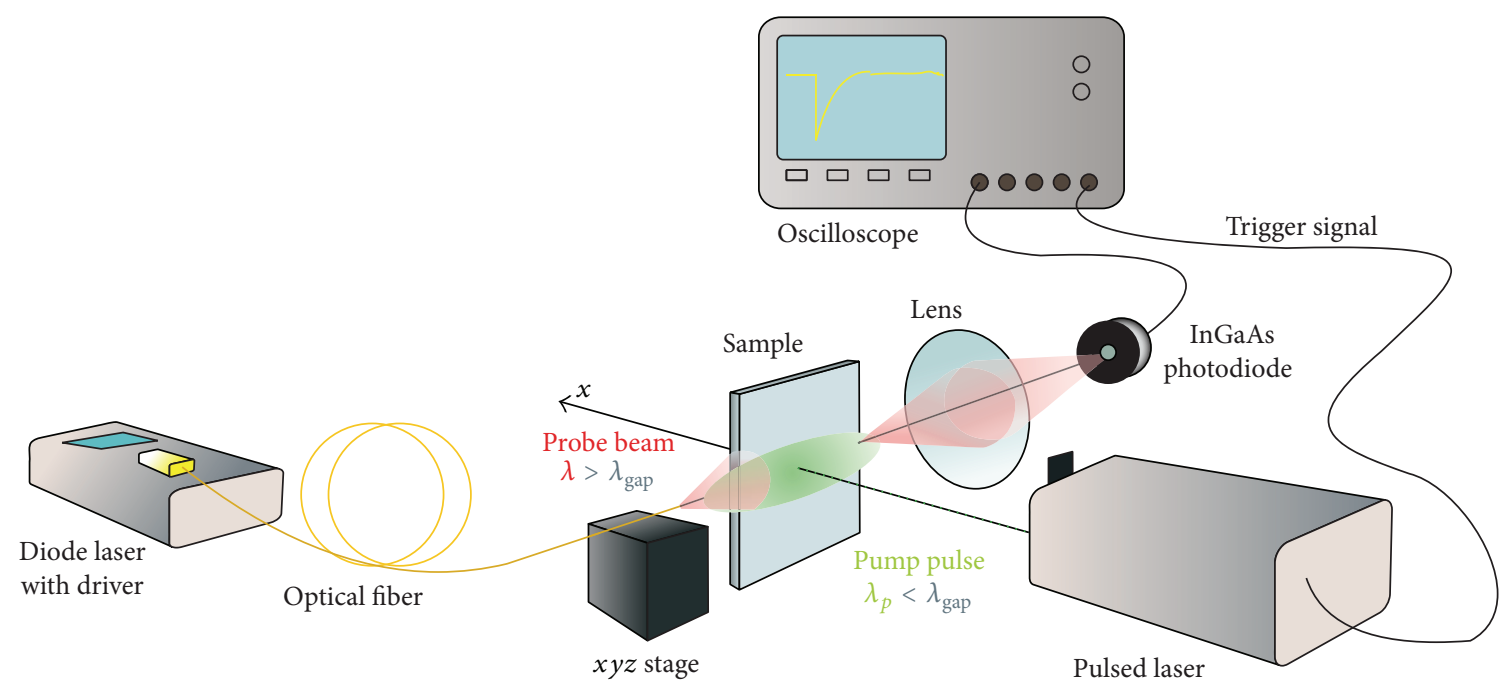

Figure 4: Pump and Probe setup in the transverse configuration. The pump pulsed beam impinges on the sample surface, while the probe beam is orthogonal and propagates inside the sample.

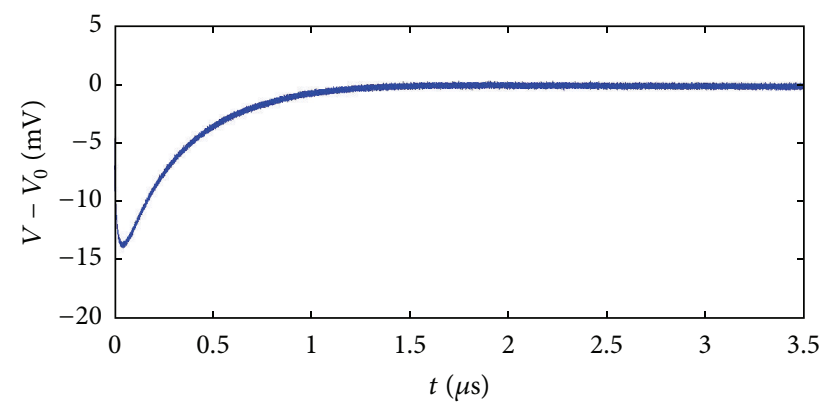

FIGURE 5: A typical signal of TPP. The attenuation of the probe beam can be observed just after the turn off of the pump pulse (the signal is normalized to the continuous level).

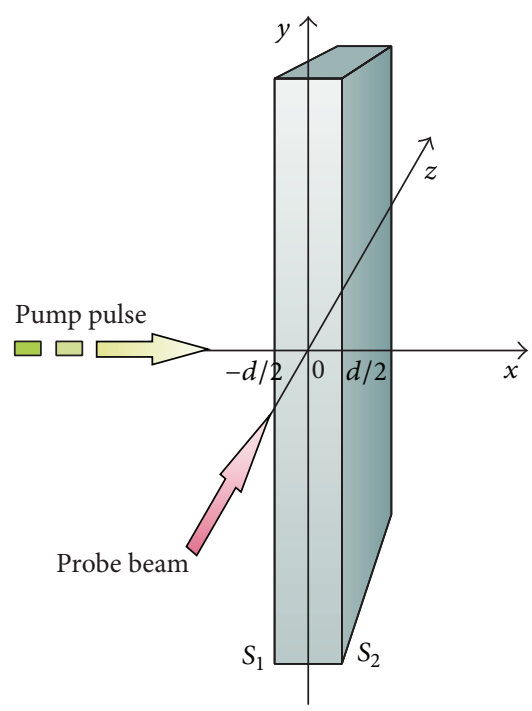

FIGURE 6: Coordinate system and wafer position adopted for the analysis of the interaction of a laser pulse with a silicon wafer.
If the duration of the pump pulse is much shorter than the expected recombination lifetime, the analysis can be carried out for an ideal delta-pulse $G(t)=N_{0} \delta(t)\left(N_{0}\right.$ is the number of photons and $\delta(t)$ the unitary Dirac distribution) and then it can be easily extended to the other cases $[52,55]$. Moreover, let us consider a pulse beam with a uniform spot size impinging on the sample surface along the propagation direction of the probe beam and similarly a probe spot size on the sample thickness. In this condition the interaction volume between the pump and the probe is extended to the whole thickness (we are approximating the interaction volume to a parallelogram) and the distribution of the excess free carriers inside it can be considered changing only along the propagation direction of the pump, denoted with $x$. The distribution of the excess free carriers generated along the wafer thickness for time $t>0$, in low injection regime and neglecting the electric field, can be considered governed by the 1-D diffusion equation

$$
\frac{\partial n(x, t)}{\partial t}=D \frac{\partial^{2} n(x, t)}{\partial x^{2}}-\frac{n(x, t)}{\tau_{b}}
$$

with the boundary conditions

$$
\begin{gathered}
D\left[\frac{\partial n(x, t)}{\partial t}\right]_{x=-d / 2}=S_{1} n\left(-\frac{d}{2}, t\right), \\
-D\left[\frac{\partial n(x, t)}{\partial t}\right]_{x=d / 2}=S_{2} n\left(\frac{d}{2}, t\right)
\end{gathered}
$$

and initial condition given by the multiple reflections of the pulse along the sample thickness, like in the Fabry-Perot cavity,

$$
n(x, 0)=N_{0}(1-R) \alpha \frac{e^{-\alpha(x+d / 2)}+R e^{-\alpha d} e^{\alpha(-x+d / 2)}}{\left(1-R^{2} e^{-2 \alpha d}\right)},
$$


with $R$ and $\alpha$ being the reflection coefficient on the wafer surfaces and the silicon absorption coefficient at the pump wavelength, respectively.

The most general solution of (12) can be expressed as

$$
n(x, t)=\sum_{k=1}^{\infty} e^{-\left(1 / \tau_{b}+a_{k}^{2} D\right) t}\left(A_{k} \cos a_{k} x+B_{k} \sin a_{k} x\right),
$$

where the coefficients $a_{k}, b_{k}$, and $B_{k}$ can be found using boundary conditions (13) and initial condition (15), respectively. In particular the coefficients $a_{k}$ are the solutions of the characteristic equation

$$
a_{k} d=\arctan \frac{S_{1}}{a_{k} D}+\arctan \frac{S_{2}}{a_{k} D}+k \pi,
$$

while, if

$$
\begin{gathered}
z_{k}=a_{k} d, \\
g_{0}=\frac{(1-R) \alpha}{1-R e^{-2 \alpha d}}, \\
B_{k}^{\prime}=\frac{\left(4 a_{k} g_{0} \alpha\right) e^{-\alpha d / 2}}{\left[b_{k}^{2}\left(a_{k} d+\sin a_{k} d\right)+\left(a_{k} d-\sin a_{k} d\right)\right]\left(\alpha^{2}+a_{k}^{2}\right)},
\end{gathered}
$$

it results in

$$
\begin{aligned}
& b_{k} \equiv \frac{A_{k}}{B_{k}}=-\frac{a_{k} D \cos z_{k}+S_{1} \sin z_{k}}{a_{k} D \sin z_{k}-S_{1} \cos z_{k}}, \\
& B_{k}=B_{k}^{\prime}\left[b_{k}\left(\cos z_{k} \sinh \frac{\alpha d}{2}+\frac{a_{k}}{\alpha} \sin z_{k} \cosh \frac{\alpha d}{2}\right)\right. \\
&\left.\times\left(1+R e^{-\alpha d}\right)\right] \\
&+B_{k}^{\prime}\left[\left(\frac{a_{k}}{\alpha} \cos z_{k} \sinh \frac{\alpha d}{2}-\sin z_{k} \cosh \frac{\alpha d}{2}\right)\right. \\
&\left.\times\left(1-R e^{-\alpha d}\right)\right] .
\end{aligned}
$$

Since the measurement determines the absorption due to all the free carriers generated along the excited volume of the sample by the pump pulse, we have to consider the average of the free carriers in that volume (at the beginning of the section we have called it interaction volume $V_{\text {int }}$ ):

$$
n_{\mathrm{av}}(t)=\frac{1}{V_{\mathrm{int}}} \int_{V_{\mathrm{int}}} n(x, t) d V
$$

with $A_{\text {int }}$ being the section of the interaction volume in the horizontal plane. The latest approximation in (20), as pointed out at the beginning of the section, subsists considering a pump pulse that uniformly illuminates the sample along the propagation direction of the probe beam. This condition can be easily obtained experimentally by means of suitable optics, like a cylindrical lens.

If the diameter of the probe beam is larger than the sample thickness, in a way that the uniform illumination of the sample cross section is assured, the interaction volume is extended to the whole sample thickness, so that, since the excess free carriers distribution depends only by the spatial coordinate $x$ along which the pump pulse is propagating, the expression (20) becomes

$$
\begin{aligned}
n_{\mathrm{av}}(t) & \propto \int_{-d / 2}^{d / 2} n(x, t) d x \\
& =\sum_{k} B_{k} b_{k} I_{k}\left(-\frac{d}{2}, \frac{d}{2}\right) u_{k}(t) \\
& =\sum_{k} B_{k} b_{k} \frac{\sin z_{k}}{z_{k}} u_{k}(t),
\end{aligned}
$$

where we have defined

$$
\begin{gathered}
u_{k}(t)=e^{-\left(1 / \tau_{b}+a_{k}^{2} D\right) t}, \\
\phi_{k}(x)=b_{k} \cos a_{k} x+\sin a_{k} x, \\
I\left(x_{i}, x_{f}\right)=\int_{x_{i}}^{x_{f}} \phi(x) d x .
\end{gathered}
$$

In the more general case of Gaussian pump pulse centered at instant $T_{0}$ and with width $\sigma$,

$$
G_{g}(t)=\frac{N_{0}}{\sqrt{2 \pi} \sigma} e^{-\left(t-T_{0}\right) / 2 \sigma^{2}} ;
$$

$n_{\mathrm{av}}$ can be derived from the convolution of $n_{\mathrm{av}}$ given by (20) with the pulse

$$
\begin{aligned}
n_{\mathrm{gav}} & =\int_{0}^{t} n_{\mathrm{av}}(\tau) G_{g}(t-\tau) d \tau \\
& =\sum_{k} B_{k} b_{k} I_{k}\left(-\frac{d}{2}, \frac{d}{2}\right) E_{k}(t),
\end{aligned}
$$

where

$$
\begin{aligned}
E_{k}(t)= & \int_{0}^{t} u_{k}(\tau) G_{g}(t-\tau) d \tau \\
= & u_{k}(t) e^{F_{k}\left(\left(\sigma^{2} / 2\right) F_{k}+T_{0}\right)} \\
& \times\left[\operatorname{erf}\left(\frac{\sigma}{\sqrt{2}} F_{k}+\frac{T_{0}}{\sqrt{2} \sigma}\right)-\operatorname{erf}\left(\frac{\sigma}{\sqrt{2}} F_{k}-\frac{t-T_{0}}{\sqrt{2} \sigma}\right)\right]
\end{aligned}
$$

with

$$
F_{k}=\frac{1}{\tau_{b}}+a_{k}^{2} D .
$$

The semilog plot of $n_{\text {gav }}(t)$ allows for defining an instantaneous observed lifetime $\tau_{0}$ given by

$$
\frac{1}{\tau_{0}}=-\frac{d}{d t} \ln \left[n_{\mathrm{gav}}(t)\right]=\frac{1}{\tau_{b}}+R_{s}(t),
$$

where, from (20), (24), and (25) it is clear that $R_{s}$ is a time dependent ratio between two series and it depends on 
the physical wafer parameters, $d, \tau_{b}, D, S_{1}$, and $S_{2}$ (see also (17)). Nevertheless it can be demonstrated [52, 55] that its asymptotic value is a constant:

$$
R_{s}(t) \stackrel{t \rightarrow \infty}{\longrightarrow} R_{s a}=a_{1} D .
$$

The previous expression shows that the asymptotic value of the inverse of the instantaneous observed lifetime can be expressed as the sum of two independent terms:

$$
\frac{1}{\tau_{0 a}}=\frac{1}{\tau_{b}}+R_{s a}=\frac{1}{\tau_{b}}+\frac{1}{\tau_{s 1}},
$$

the first of which is equal to the inverse of the bulk lifetime and the second one is independent of latest and dependent on the surface effects, as $a_{1}$ depends on $S_{1}, S_{2}$, and $d$ (the subscript $s$ refers to this dependence of the asymptotic value of $R$ ).

\section{TPP Parameter Extraction}

The relation (29) is the core of the TPP method. In accordance with the sample properties and the set-up configuration, different information can be extracted by the measurement. In this way the TPP presents several versions.

6.1. Single Slope Method. When the surface effects can be neglected or when $\tau_{s 1}$ is known, from (29) the bulk lifetime can be directly derived. Luke and Cheng [55] plotted $\tau_{s 1}$ versus the wafer thickness $d$ for different values of the surface recombination velocity (from 10 to $10^{6} \mathrm{~cm} / \mathrm{s}$ ), supposing $S_{2}=$ $S_{1}$, and showed that, for $S>10^{5}$, when $d$ increases, the value of $\tau_{s 1}$ does not change. Really the product $S d$ is determinant, since when it increases, the root $a_{1} d$ of the transcendental equation (17) approaches a limit value. This suggests that for $S d \rightarrow \infty$ the surface effects can be neglected and the bulk lifetime can be easily derived as

$$
\tau_{b}=\frac{\tau_{0 a} \tau_{s 1}}{\tau_{s 1}-\tau_{0 a}},
$$

with $\tau_{s 1}=1 / a_{1} D$ being analytically computed and $\tau_{0 a}$ derived from the experimental data. The simulations reported in [55] show that in practice the influence of $S$ can be neglected for Sd $>120 \mathrm{~cm}^{2} / \mathrm{s}$.

6.2. Dual Slope Method. If the surface effects cannot be neglected, two values of the instantaneous observed lifetime can be used to derive from the experimental data the bulk lifetime and the surface recombination velocities: the asymptotic value (defined in (29)), and the maximum value (defined in the same manner, where obviously $R_{s}(t)$ is replaced with its maximum value $\left.\max \left[R_{s}(t)\right] \equiv R_{s m}\right)$.

In this way, if $S_{2}=S_{1}=S$, to determine the two unknown quantities $\tau_{b}$ and $S$, we have a system with two equations:

$$
\begin{gathered}
\frac{1}{\tau_{0 a}}=\frac{1}{\tau_{b}}+R_{s a}, \\
\frac{1}{\tau_{0 m}}=\frac{1}{\tau_{b}}+R_{m}
\end{gathered}
$$

so that

$$
\left(R_{s m}-R_{s a}\right)_{\mathrm{anal}}=\left(\frac{1}{\tau_{0 m}}-\frac{1}{\tau_{0 a}}\right)_{\mathrm{exper}}
$$

can be used to evaluate $S$ and

$$
\tau_{b}=\frac{\left(\tau_{0 a}\right)_{\mathrm{anal}}}{1-\left(R_{s a}\right)_{\text {exper }}\left(\tau_{0 a}\right)_{\mathrm{anal}}}
$$

gives $\tau_{b}$. The subscripts "anal" and "exper" indicate that the quantities are analytically computed or experimentally determined. Luke and Cheng wisely suggest that to derive $\tau_{b}$ it is better to use $\tau_{0 a}$, since it can be better determined and that the error computed to neglect the dependence of $R_{s}$ on $\tau_{b}$ is about 0.1 per cent over the range of $S=1$ to $10^{6} \mathrm{~cm} / \mathrm{s}$, or rather for $40 \leq S d \leq 10^{6} \mathrm{~cm}^{2} / \mathrm{s}$. This shows the complementary of the single slope and dual slop methods.

6.3. Two Wafer Method. In the region not covered by the previous two methods, Luke and Cheng proposed to utilize the $R_{s}$ dependence on the wafer thickness. In fact using two wafers which differ only in thickness, $d_{1}$ and $d_{2}$, the former system (33) can be replaced with

$$
\begin{aligned}
& \left(\frac{1}{\tau_{0 a}}\right)_{1}=\frac{1}{\tau_{b}}+\left(R_{s a}\left(d_{1}, S, D\right)\right)_{1}, \\
& \left(\frac{1}{\tau_{0 a}}\right)_{2}=\frac{1}{\tau_{b}}+\left(R_{s a}\left(d_{1}, S, D\right)\right)_{1} .
\end{aligned}
$$

Really the method works well even for large value of $S d$, so that more than complementary to the previous ones, can substitute them. Nevertheless Irace et al. [59] observed that since the two measurements of $\tau_{0}$ are done on the same material and, at most, averaged on an area of about $1 \mathrm{~cm}^{2}$, equal to the probe beam spot, it can be safely assumed that the bulk recombination lifetime remains unchanged. On the other hand, since the separation and calculation of the surface effects rely on the hypothesis that also SRV has to remain unchanged, there is the problem of assessing a surface treatment procedure that is well reproducible and sets equal surface recombination velocities on both sides of each sample and in both samples. These considerations led them to perform an analysis of the error sources of this procedure and of the uncertainties that they can lead to. They showed the effect of different values of SRVs on the two measurements using a graphical approach. Therefore, for each measured value of the instantaneous lifetime, it is possible to draw a line on the $\tau_{b}-S$-plane that, of course, will span all the allowed values of $\tau_{b}$ and $S$. On the other hand, because two measurements are available, the two solutions intersect in a single point, corresponding to the looked for $\left(\tau_{b}, S\right)$ pair. If there are uncertainties $\Delta \tau_{i}(i=1,2)$ on the quantities, a region can be identified in the plane, bounded by the lines $\tau_{01} \pm \Delta \tau_{1}$ and $\tau_{02} \pm \Delta \tau_{2}$, respectively, for each sample. Intersecting these two regions, the area where the solution of the (34) lies can be identified. It contains the information about the uncertainty on the surface recombination velocity 


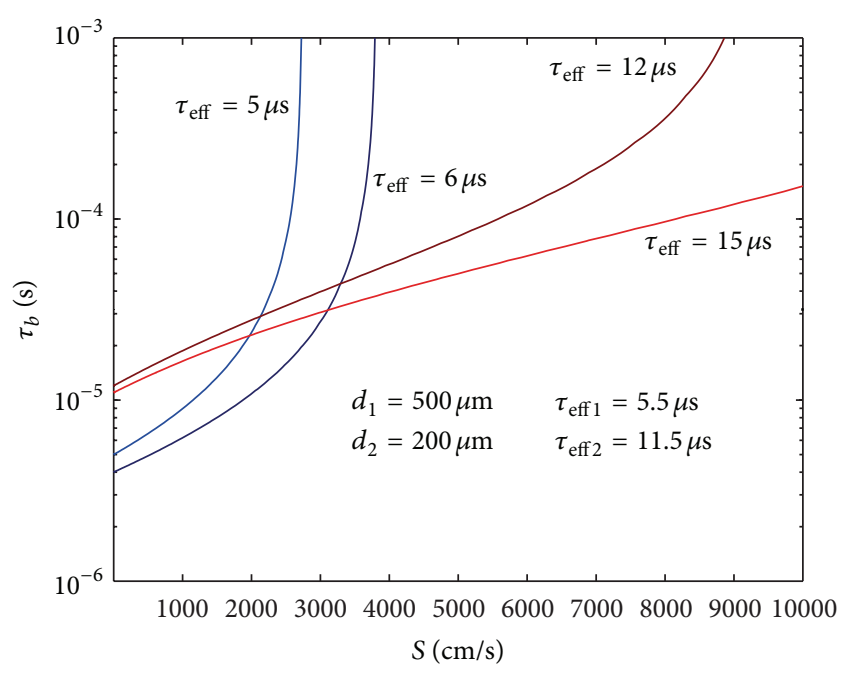

FIGURE 7: $\tau_{b}-$ SRV plane as reported in [59].

or can take into account the effects of the experimental uncertainties on the measured $\tau_{0}$ value. An example of the above procedure is given in Figure 7.

6.4. Dual Slope Method on Both Surface. If $S_{0} \neq S_{1}$ a further equation is needed to determine all the three parameters. Kousik et al. in 1991 [52] proposed to perform the measurement flipping the wafer, to have one measurement with the pump beam impinging on the wafer surface with recombination surface velocity $S_{1}$ and one complementary measurement with the pump impinging on the other surface.

The available equations are thus

$$
\begin{aligned}
\frac{1}{\tau_{0 m}}=\frac{1}{\tau_{b}}+R_{m} \quad \text { for }\left(S_{1}, S_{2}\right) \quad \text { or } \quad\left(S_{2}, S_{1}\right), \\
\frac{1}{\tau_{0 a}}=\frac{1}{\tau_{b}}+R_{s a} \text { for }\left(S_{1}, S_{2}\right), \\
\frac{1}{\tau_{0 a}}=\frac{1}{\tau_{b}}+R_{s a} \text { for }\left(S_{2}, S_{1}\right),
\end{aligned}
$$

where the notation $\left(S_{i}, S_{j}\right)(i \neq j)$ means that the pump impinges on the surface $S_{i}$.

6.5. Multiwavelength Method. The bulk lifetime and the surface recombination velocity do not depend on the pump wavelength but from (27) we can see that the instantaneous observed lifetime $\tau_{0}$ depends on it. In particular, the dependence is all included in the initial part, while the asymptotic part is unaffected. This means that, for $t \rightarrow \infty, \ln \left(n_{\mathrm{av}}(\lambda, t)\right)$ has the same slope $1 / \tau_{0 a}$ (see (24)-(29)). In other words, the asymptotic separation, between two decay curves relative to two different pump wavelengths $n_{\mathrm{av}}\left(\lambda_{1}, t\right)-n_{\mathrm{av}}\left(\lambda_{2}, t\right)$, approaches a constant value. This observation suggested to Buczkowski and coworkers [62] in 1991 (when the dual slope method was not proposed yet) that the surface effects can be extracted from this value. They verified the idea with a different technique, called two-laser microwave reflection, in the hypothesis of equal surface recombination velocities. The technique uses the reflection or absorption of a microwave radiation on wafer surface as probe signal.

From 2002 to 2005 Irace and coworkers [63-68] transposed and tested the idea with a pump and probe setup, using a laser diode @1550 nm as probe beam and the fundamental and the second harmonic of a $7 \mathrm{~ns}$ pulsed Nd-YAG laser $\left(\lambda_{1}=\right.$ $1064 \mathrm{~nm}$ and $\lambda_{2}=532 \mathrm{~nm}$ resp.) to pump the sample.

Defining

$$
\delta_{a}=\lim _{t \rightarrow \infty} \delta(\Delta \lambda, t)=\ln \left(\frac{N_{\text {rel }}\left(\lambda_{1}, t\right)}{N_{\text {rel }}\left(\lambda_{2}, t\right)}\right)
$$

with $N_{\text {rel }}=n_{\mathrm{av}}(\lambda, t) / n_{\mathrm{av}}(\lambda, 0)$, and $\Delta \lambda=\lambda_{1}-\lambda$, it is clear that (36) is a function of $\Delta \lambda, D, d, S_{1}, S_{2}$.

If $S_{1}=S_{2}=S$, the experimental determination of $\delta_{a}$ for a fixed pair of wavelength, is enough to determine $S$. Successively, $\tau_{b}$ can be determined by means of (29).

For any pair of wavelengths, $\delta$ is an increasing function of $S$. For small value of $S$, where the surface effects are negligible with respect to the bulk ones, it is difficult to distinguish between two cases with different absorption; therefore, the values assumed by $\delta$ are small. When $S$ increases, the separation between the decay curves increases, reaching a saturation value for high $S$. This limits the applicability of the method to $S<10^{5}$, while the lower limit is determined by the signal-to-noise ratio.

If $S_{1} \neq S_{2}$, having only two pump wavelengths, $\lambda_{1}$ and $\lambda_{2}$, it is possible to determine both the surface recombination velocities by means of measurements with the pump impinging one time on one of the wafer surface and another time with the pump impinging on the other wafer surface, like in the dual slope method. In this manner $S_{1}$ and $S_{2}$ can be determined from the experimental decay curve solving the system

$$
\begin{aligned}
& \left(\delta_{a 12}\right)_{\text {exper }}=f\left(\Delta \lambda ; S_{1}, S_{2}\right) \text { for pump on surface } 1 \\
& \left(\delta_{a 21}\right)_{\text {exper }}=g\left(\Delta \lambda ; S_{2}, S_{1}\right) \text { for pump on surface } 2
\end{aligned}
$$

(with obvious meaning of the subscripts 12 and 21). Then $\tau_{b}$ is computed using (33), with $R_{s} a$ relative to one of the two measurements.

The main advantage of the technique is that it gives the values of $S_{1}, S_{2}$, and $\tau_{b}$ even when the sample thickness is comparable to the minority carriers diffusion length, for which the effects of recombination at the two interfaces are coupled.

6.6. Cross-Sectional Profiling Technique. The previously exposed methods are based on the assumption that the other parameters of the sample are well known (see, e.g., (17) and (28), (29), depending on $D$ ) but it could not be the real case and, moreover, some other experimental variables could not be exactly controlled. Mathematically this means that the used equation systems require additional equations to be solved.

Moreover they work in conditions such that the interaction volume between the pump and probe is wide as the whole 
sample thickness. In this condition, depending on the sample thickness and on the diffusion length, the bulk contribution to the recombination lifetime could be dominant respect to the surface contribution or vice versa. To overcome these difficulties Gaubas et al. [69, 70] proposed an alternative method based on the simultaneous analysis of the time and amplitude characteristics of the excess free carriers decay by scanning the sample thickness with a probe beam spot size smaller than the wafer thickness. In this way the interaction volume between the pump (illuminating the whole sample along the propagation direction of the probe) and the probe is limited to a narrow portion along the sample thickness. They showed (with simulation and experimental results) that the transient decay shape changes with the depth along the sample thickness, with that meaning, as the distance of the probe beam from the pump incident surface on the sample increases. In particular they observed that the decay shows concavity change, which passes to concave to convex when the depth approaches the middle of the sample. This happens because the surface effects decrease and the observed fraction of the excess free carriers in that region is more governed by the bulk recombination. The same behavior is observed without the scanning but changing only the probe beam spot size.

Recently an improved version of the technique has been reproposed, where a detailed analysis of different probe beam shapes has been evidenced (i.e., both the case of collimated and divergent probe beam) $[71,72]$. The improvement is the combination of it with the multiwavelength method. In the particular case the pump is a tunable source in the range 500-1150 nm, constituted by an Optical Parametric Oscillator (OPO) pumped with a third harmonic of a ND:YAG laser. In this way the method results more robust and accurate since it has both the advantages of of the cross-sectional profiling technique and of the multiwavelength one.

About the latest it must be observed that a tunable source allows for having more than two wavelengths to perform the measurements, so that instead of the system (37), relations like (36) are used for each pair of wavelengths. This avoids the measurements with the pump impinging one time on one surface and one time on the other surface, which can lead to some errors. Moreover the desired quantities are better and more accurately retrieved by fitting simultaneously a set of equations similar to (27), obtained, nevertheless, with a more accurate theoretical model of the best fit curve. The measurement is then performed from the a-priori knowledge of the other sample parameters involved, and allows for a better the determination of them.

At the beginning of this section, we stated that the parallel configuration is more suitable for wafer lifetime mapping (see, e.g., [56]); nevertheless, it must be observed that the lifetime mapping cannot be done with a unique acquisition. The sample must be scanned, so that, depending on the pump pulse frequency, on the probe beam spot size and sample dimension the measurement requires an amount of time that typically is at least of order of minutes. For a more efficient and advantageous lifetime mapping an array of detectors can be used to photograph the whole sample in one time. The technique that we present in the next sections allows exactly for this.
Moreover, it must be noted that in the transient lifetime measurements could happen that the effective measured lifetime can differ from the actual lifetime by a factor equal to 2 or 3 [61]. This inconvenience is not present in the steadystate techniques. We will understand this in the next section, before presenting the ILM technique.

\section{Differential and Actual Lifetime}

In the paper we have several times mentioned that the recombination parameters in general depend on the excess carriers concentration, thus on the injection level. When the injection level is high, that is, when the generated excess carriers concentration $\Delta n$ is higher than the majority carriers concentration, really the observed transient decay is not a single-exponential and should require, for data extraction, a more complicated treatment. Nevertheless Aberle et al. [7376] acutely noted that the situation can be treated in small signal regime. They suggested that it can be actuated adding a constant bias of suitable intensity to the pulse pump to generate a constant background carriers concentration, $n_{b}$, a lot higher than the excess carriers concentration induced by the pump pulse, $n_{p}(t)$. So that the excess carriers can be written as

$$
\Delta n(x, t)=n_{b}(x)+n_{p}(x, t) .
$$

In this way they showed that the measured lifetime is not the actual but a differential lifetime related to the former by the relation

$$
\tau\left(n_{b}\right)=\frac{1}{n_{b}} \int_{0}^{n_{b}} \tau_{\text {diff }} d \Delta n
$$

with a similar expression for the surface recombination velocity. The differential and the actual lifetime are equal only in the particular case of a constant bulk lifetime. This situation is often verified in the case of low-injection condition. At high-injection conditions, when for example the Auger recombination becomes dominant, it can be easily demonstrated that the small signal approach predicts a value of the differential lifetime three times smaller than the actual one. For the radiative recombination the reduction factor is 2 [76].

The steady-state methods, where the generation rate and the excess carriers density are controlled simultaneously, present the advantage to measure directly the actual lifetime. In fact, in the steady-state conditions, since the generation rate and the recombination rate are equal and proportional to the power density of the bias light, on the approximation that the excess carriers concentration is approximately constant throughout the sample, the result is [75]

$$
\tau\left(n_{b}\right)=\frac{1}{P} \int_{0}^{P} \tau_{\text {diff }}(P) d P .
$$

Consequently, as the integration is performed by the detector, the actual lifetime is directly measured. This is a further advantage of the ILM method, which is detailed in the next sections. 


\section{Infrared Camera Lifetime Mapping/Carrier Density Imaging}

The Infrared Lifetime Mapping (ILM) method was presented the first time in 2000 by Bail et al. [2]. Subsequently in 2001 Riepe and coworkers [3] presented a variation on the theme called Carrier Density Imaging (CDI), improved with the lock-in detection technique [77].

The method is also based on the free carriers absorption. Like in the pump and probe method, the sub-bandgap radiation is used as a probe propagating through the sample, to monitor the absorption variation (or rather, the transmittance variation) due to the free carriers generated by the above band gap pump photons. Nevertheless, a black body radiation is used as probe instead of a laser. This radiation, belonging to the $5-25 \mu \mathrm{m}$ range of the IR spectrum, is partially transmitted by the silicon wafer and can be detected by an infrared CCD (charge coupled device) camera, like the ones used in IR thermography setups $[77,78]$. So that, when the sample is not excited by the laser pump beam (pump off), the CCD camera returns an image with a reference intensity level, $S_{\text {off }}$. This image corresponds to the currents intensities produced by each pixel sensor illuminated by the black body infrared radiation that had passed through the silicon wafer. In others words, it is a matrix of $M \times N$ elements, $S_{i j}$, as many as the number of CCD pixels. When the pump impinges on the sample (pump on), the absorption of photons with energy greater than the energy band gap generates an excess of free carriers (hole-electron pairs) that modifies the absorption coefficient and consequently causes an intensity variation of the transmitted black body radiation. The camera snapshot reflects this variation with a different intensity currents map $S_{\text {on }}$.

The authors showed that it is easy to derive the excess carriers density variation per unit area, $\Delta n W$ (where $n$ is the free carriers density and $W$ is the sample thickness) from the camera contrast

$$
K=S_{\text {off }}-S_{\text {on }},
$$

once it is calibrated, and, successively, the effective, actual lifetime. This is the reason of the adopted name for the technique, CDI, given by [3]. The effective, actual lifetime is derived by means of the steady-state solution of the diffusion equation

$$
\tau_{\text {eff }}=\frac{\Delta n W}{G},
$$

where $G$ is the generation rate, equal to the pump density photons flow per unit time (which has the dimension of $\left[\right.$ lenght ${ }^{-3} \times$ time $\left.^{-1}\right]$ ) reduced by the relative quantity reflected by the wafer incident surface

$$
G=\Phi\left(1-R_{f}\right),
$$

with $R_{f}$ being the silicon wafer front side reflectance at pump wavelength.

The ILM schematic setup is shown in Figure 8.

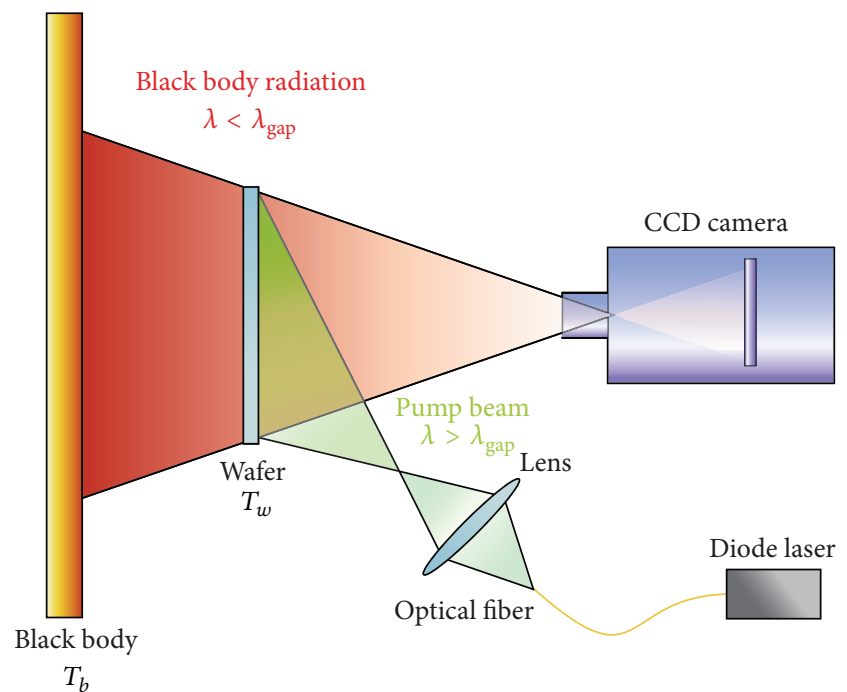

FIGURE 8: In the ILM/CDI schematic setup.

8.1. The Detected Black Body Radiation. The camera signal is proportional to the black body radiation transmitted through the wafer (of thickness $W$ and reflectance on the incident surface $R$ ) and integrated on the camera spectral range $\left[\lambda_{\min }, \lambda_{\max }\right]:$

$$
\begin{aligned}
S & =\Gamma \int_{\lambda_{\min }}^{\lambda_{\max }}(1-R)^{2} e^{-\alpha_{\mathrm{fc}}(\lambda) W} I\left(\lambda, T_{b}\right) d \lambda \\
& \approx \Gamma \int_{\lambda_{\min }}^{\lambda_{\max }}(1-R)^{2} \alpha_{\mathrm{fc}}(\lambda) W I\left(\lambda, T_{b}\right) d \lambda,
\end{aligned}
$$

where $\Gamma$ is a proportionality constant that takes into account the $\mathrm{CCD}$ response and the sample geometry;

$$
I\left(\lambda_{\mathrm{det}}, T_{b}\right)=\frac{2 \pi h c^{2}}{\lambda^{5}} \frac{1}{e^{\left(h c / \lambda k_{B} T_{b}\right)-1}} \approx \frac{\gamma}{\lambda^{5}} e^{-\Psi / \lambda T_{b}}
$$

(with $\gamma=2 \pi h c^{2}$ and $\Psi=h c / k_{B}$ ) is the black body spectral density, given by Planck's Law approximated in the case of black body temperature $T_{b}$ greater than hundreds kelvin ( $h$ is the Planck constant, $c$ is the vacuum light velocity, and $k_{B}$ is the Boltzmann constant); $R$ is the silicon wafer reflectance at the black body wavelength and, we remember, $\alpha_{\mathrm{fc}}$ is the free carriers absorption coefficient $\left(\mathrm{cm}^{-1}\right)[2,34,37-39]$.

8.2. Emission Mode. Since each body which is heated behaves like a black body, the emitted infrared radiation can be detected increasing the wafer temperature $T_{w}$ and, like in the absorption mode, the optical pumping determines a variation of the carriers intensity that leads to a variation of the emitted radiation. From the Kirchhoff Law at thermal equilibrium the emission coefficient $\varepsilon$ must be equal to the absorption coefficient $\alpha$.

In the emission mode the wafer is heated above the black body temperature, so that the black body radiation of the wafer on the background is dominant. In fact, as made explicit in [79], the camera detects a signal that is the superposition 


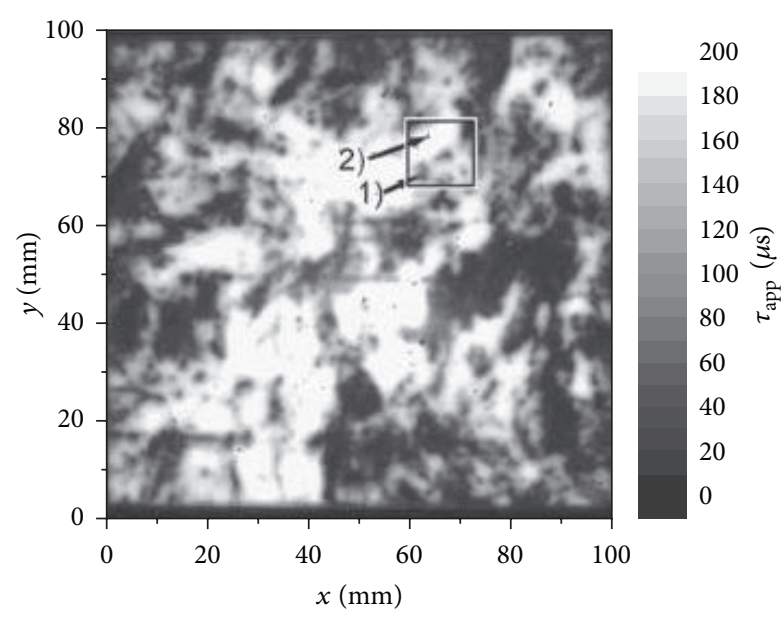

FIgURE 9: Typical ILM image as reported in [60].

of the black body radiation given by (44) and of a black body radiation emitted just by the sample, since at thermal equilibrium the free carriers also emit infrared radiation. Depending on the wafer temperature with respect to the black body temperature (and vice versa), the emission component can dominate on the transmitted one. Denoted with $\varepsilon_{\mathrm{fc}}(\lambda)$, the emissivity of the wafer due to the free carriers, the emission component of the signal can be written like in (44) using Planck's law (45) for nonideal black bodies $(\varepsilon(\lambda)<1)$ :

$$
S_{\mathrm{em}}=\Gamma \int_{\lambda_{\min }}^{\lambda_{\max }} \varepsilon_{\mathrm{fc}}(\lambda) \frac{\gamma}{\lambda^{5}} e^{-\Psi / \lambda T_{w}} d \lambda .
$$

The total signal detected by the camera is thus

$$
S=S_{\mathrm{em}}-S_{a b}
$$

where $S_{\mathrm{em}}$ and $S_{a b}$ are given by (44) and (52), respectively. It is clear now that if $T_{b} \ll T_{w}$ the emission signal dominates (in the experiments $T_{b}>350 \mathrm{~K}[2,79,80]$ ).

8.3. ILM/CDI Calibration. The calibration can be performed comparing camera image of well-characterized wafer (usually a $p$-type) that differs from a reference one only in dopant concentrations $N$ and, at the most, in the thickness $W$ (Figure 9). In fact, the plot of the camera contrast relative to each pixel individuated by the coordinates in the $x$ - $y$ plane, $K_{C}(x, y)=S_{N_{i} W_{i}}(x, y)-S_{N_{1} W_{1}}(x, y)$, obtained subtracting the image of the $i$ th wafer to that one of the reference wafer, labeled 1 , as function of the free carriers density per unit area relative to the same wafers and to the same pixel, $\Delta(N W)(x, y)=N_{i}(x, y) W_{i}-N_{1}(x, y) W_{1}$, gives the following experimental relation:

$$
K_{C}(x, y)=m \Delta(N W)(x, y),
$$

that, suitably corrected, gives the final conversion rule between the free carriers density per unit area variation and camera contrast:

$$
\Delta(n W)(x, y)=\frac{K(x, y)}{m \sigma} \kappa(x, y)
$$

The correction factor $\sigma$ takes in account the fact that only wafers with one type of dopant are used in the calibration [2], while the absorbed pump photons generate excess electrons and holes that bring about a variation in the absorbed black body radiation. This correction factor in the case of $p$-type calibration wafers must be

$$
\sigma=\left(1+\frac{\alpha_{n}}{\alpha_{p}}\right),
$$

where $\alpha_{n}$ and $\alpha_{p}$ are the absorption coefficients of the free electrons and holes, respectively, at the absorbed radiation wavelength $\lambda$ (the black body detected radiation) $[2,34,37-$ 39]. Obviously the absorption coefficients must be inverted in (50) if the calibration is performed with $n$-type wafers. The factor $\kappa(x, y)$, suggested by [81], takes into account both the individual pixel sensor responsivity (the variation of the sensor response to the variation of the incident radiation) and the CCD sensor geometrical configuration. It must be considered that the central pixels of the CCD array see the sample under a larger steradian than the pixels near the array edge [81], so that the incident IR power flow is different. The $\kappa(x, y)$ factor is specific to each CCD array comparing the pixels response respect to the response of a reference pixel.

8.4. ILM/CDI Sensitivity. To quantify the ILM/CDI sensitivity a noise equivalent lifetime (NEL) is defined $[2,81]$ as the effective minority carrier lifetime that the sample must have to generate an infrared camera contrast that equals the camera noise. This quantity is related to camera detection limit (or minimum resolvable contrast), that is, the minimum variation of the incident radiation that the camera can distinguish. For the infrared camera it is expressed in terms of the minimum appreciable variation of temperature, the socalled NETD (noise equivalent temperature difference), since they are usually used to detect the temperature dependent black body radiation, like in the ILM/CDI and thermography applications. Nevertheless, as observed in [82], the nominal value of the NEDT cannot be considered, since it is defined by measuring a black body at room temperature and using specific camera settings and specific $f$-number of the optics, while the experimental condition is usually different. It must be measured for the specific setup and its results are usually higher than the nominal one.

Using the (45), the minimum contrast can be written as

$$
\begin{aligned}
K_{d l} & =\Gamma\left(I\left(\lambda_{\mathrm{det}}, T_{b}+\mathrm{NETD}\right)-I\left(\lambda_{\mathrm{det}}, T_{b}\right)\right) \\
& \approx \Gamma \frac{\gamma}{\lambda_{\mathrm{det}}^{5}}\left(e^{-\Psi /\left(T_{b}+\mathrm{NETD}\right)}-e^{-\Psi / T_{b}}\right) \\
& \approx \Gamma \frac{\gamma}{\lambda_{\mathrm{det}}^{5}} \frac{\Psi \mathrm{NEDT}}{T_{b}^{2}} e^{-\Psi / T_{b}},
\end{aligned}
$$

where, we remember, $\gamma=2 \pi h c^{2}$ and we have redefined $\Psi=h c / k_{B} \lambda_{\text {det }}$. The latest approximation derives from the consideration that is NETD $\ll T_{b}$ (for state-of-art cameras NEDT $\sim 10 \mathrm{mK}$ ).

When the camera ILM/CDI signal equals the minimum camera contrast, then $\tau_{\text {eff }}=$ NEL. Neglecting the reflectance 
(in the experiment it is possible using an antireflection coating on the back of the wafer), supposing that the radiation is optically filtered at wavelength $\lambda_{\text {det }}$ (as often it is in practice), we can approximate (44) as

$$
S \approx \Gamma \beta \Delta n W \frac{\gamma}{\lambda_{\text {det }}^{3}} e^{-\Psi / T_{b}},
$$

so that from (42), (43), (52), and (51), we obtain

$$
\mathrm{NEL}=\frac{h c}{k_{B} T_{b}^{2} \lambda_{\text {det }}^{2}} \frac{1}{\beta \Phi\left(1-R_{f}\right)} \frac{\mathrm{NETD}}{\sqrt{N}},
$$

where $N$ is the number of averaged images $[2,81,82]$.

The sensitivity of the ILM/CDI in the emission mode can be derived in the same manner, obtaining at the end an expression similar to the previous one where $T_{w}$ appears instead of $T_{b}$.

Equation (53) shows that the setup sensitivity increases

(i) when the black body temperature in the absorption mode and the wafer temperature in the emission mode increase. This suggests that the latest mode results are more sensitive. In fact in accordance with the Kirkoff Law, at thermal equilibrium the absorbed infrared radiation is equal to the emitted infrared radiation, but, in spite of the smaller emissivity of the wafer with respect to the quasi-ideal black body used, the dependence of the black body radiation on temperature must be considered. Therefore, while relatively intense signals are reached in emission mode heating the wafer, in the other configuration the black body must be cooled, determining a reduction of the emitted infrared intensity;

(ii) when a detection wavelength around $8 \mu \mathrm{m}$ is used. This wavelength corresponds to the maximum of the black body spectral emission. Cameras with the maximum response in the MIR must be chosen;

(iii) with the increasing of the camera sensitivity (decreasing of the NEDT) and camera speed (the camera frame rate increases and thus the number of averaged images, or rather the averaging time). The introduction of the lock-in technique [77], already suggested in [2] and tested the first time in the CDI apparatus by Riepe et al. [3], allows for reducing the NEL by 2 orders of magnitude.

8.5. Lateral Resolution. The lateral resolution of the ILM depends on the optical set up and on the camera resolution. With a $384 \times 288$ pixel array detector and an optics that allows for focusing on an area varying between $15 \times 15100 \times 100 \mathrm{~mm}$, the lateral resolution (given by the ratio between the focused area and the number of lateral pixels) of about 50 and $350 \mu \mathrm{m}$, respectively, can be reached [81]. With the modern camera, increasing the number of pixel, and with different optics, the resolution can be increased to few tens of microns.

\section{Dynamic Carrier Lifetime Imaging}

We have noted that, being the ILM a steady-state technique, one of its disadvantages is the necessity of an accurate calibration. Ramspeck and coworkers recently developed a dynamic version of the technique (dynamic-ILM) [61] that presents the advantages of the transient technique to avoid the calibration and the advantages of the ILM to yield a very fast and high spatially resolved data acquisition.

The setup is similar to the common ILM technique but the applied excitation source is a square-wave-shaped illumination and it implements a lock-in technique for the data processing (this not only to improve the signal-to-noiseratio, as we will see). The pump period is chosen such that the sample reaches a stationary condition in the semiperiod when the pump is on. In this way four images are recorded: the first one immediately after switching on the pump; the second when the steady-state condition is achieved; the third immediately after switching off of the pump; and the fourth when the new steady state is achieved with the pump off. The latest image records the background illumination that must be used to correct the other images. If the camera integration time is chosen sufficiently short, that means of the order of the sample expected effective lifetime, $t_{\text {int }} \approx \tau_{\text {eff }}$, the first and the third images record the transient rise and decay of the excess free carriers and, in this condition, a sufficient contrast between the signals relative to the the images of the transients respect the signal relative to the image of the steady-state is achieved.

In Figure 10 the illustration of the images acquisition timing with respect to the pump illumination is reported, as shown in the original work [61].

The authors observe that the ratio between the background corrected images relative to the steady-state and to the rise transient, respectively, (the first image and the second one) depends only on $t_{\text {int }}$ and $\tau_{\text {eff }}$, so that, known the former, the second one can be evaluated without requiring further calibration. Nevertheless, using the lock-in detection the correction of the background results are automatic. In fact, the acquisition is composed by two subsequent steps: in the first one the four images recorded during one excitation period are multiplied with coefficient of a sine and a cosine function, in phase with the excitation; in the second one the four images are summed up. The results are the cosinecorrelation function, $S_{\text {cos }}$, and the sine-correlation function, $S_{\text {sin }}$, that give, respectively, the difference between the first and the third images, and between the second and the fourth images:

$$
\begin{aligned}
S_{\text {cos }}= & \int_{0}^{t_{\text {int }}} \Delta n(t) d t-\int_{T / 2}^{T / 2+\text { int }} \Delta n(t) d t \\
= & S_{\text {st-st }}\left\{t_{\text {int }}-2 \tau_{\text {eff }}\left[1-\exp \left(-\frac{t_{\text {int }}}{\tau_{\text {eff }}}\right)\right]\right\} \\
S_{\text {sin }}= & \int_{T / 4}^{T / 4+t_{\text {int }}} \Delta n(t) d t-\int_{3 T / 4}^{3 T / 4+\text { int }} \Delta n(t) d t \\
= & S_{\text {st-st }} \\
& \times\left\{t_{\text {int }}-2 \tau_{\text {eff }}\left[\exp \left(-\frac{T}{4 \tau_{\text {eff }}}\right)-\exp \left(-\frac{T+4 t_{\text {int }}}{4 \tau_{\text {eff }}}\right)\right]\right\},
\end{aligned}
$$




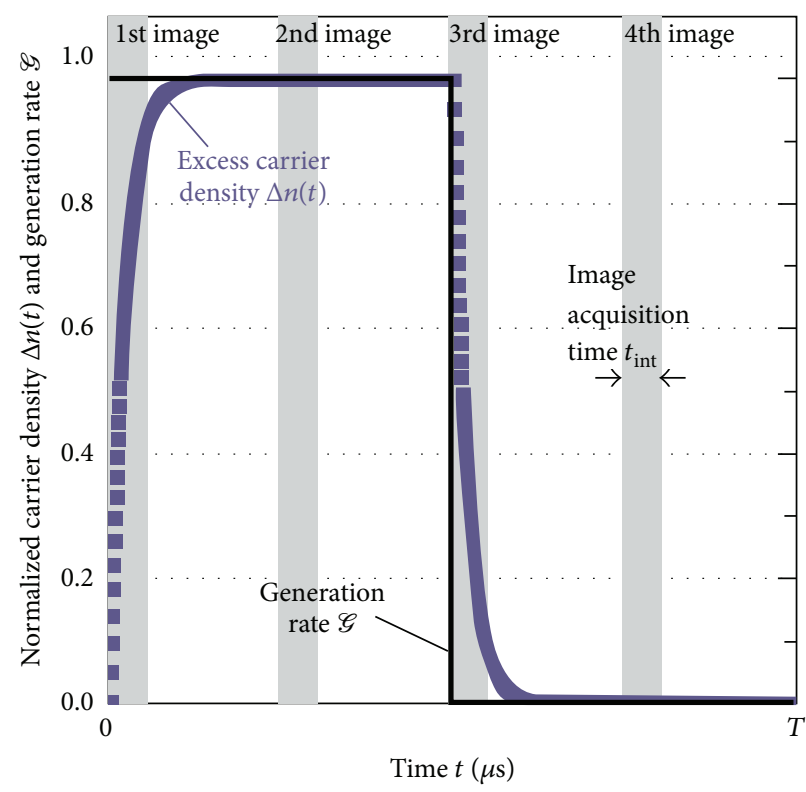

(a)

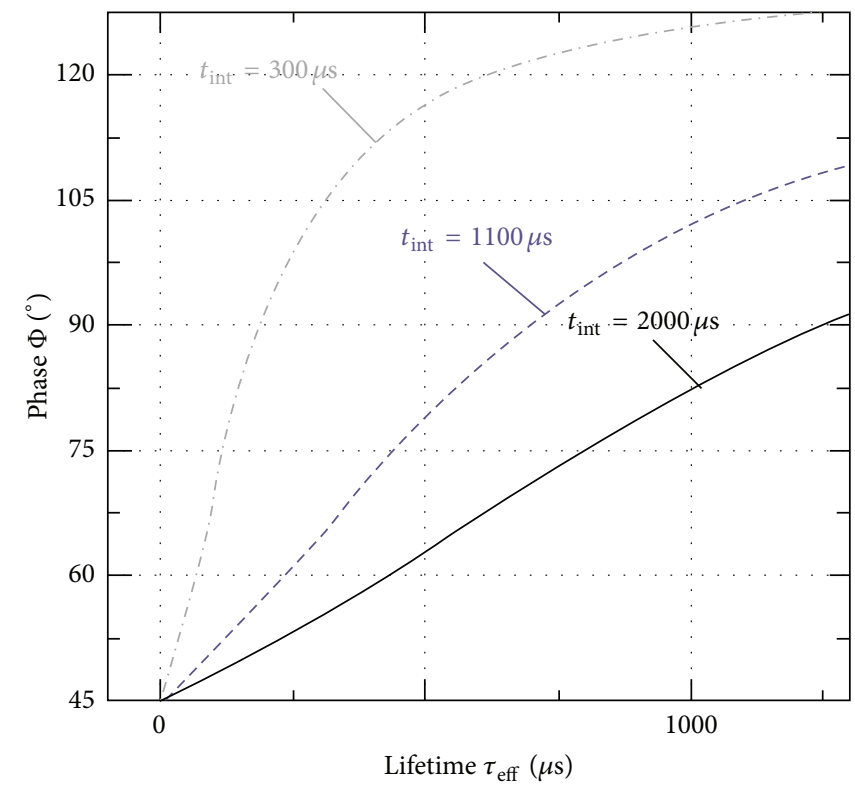

(b)

Figure 10: (a) Schematic of the generation rate $\mathscr{G}$, excess carriers density $\Delta n(t)$, and image acquisition during one measurement period of length $T$, as reported in the original work of Ramspeck et al. [61]. (b) Relationship between lifetime $\tau_{\text {eff }}$ and phase lock-in phase $\Phi$ for several image acquisition times as reported in the original work of Ramspeck et al. [61].

where $S_{\text {st-st }}=\mathscr{G} \tau_{\text {eff }}$ represents the steady-state signal that would be recorded if any background would be present, while $T$ is the lock-in period. The authors observe that the lockin phase, defined as $\Phi=\arctan \left(S_{\text {sin }} / S_{\text {cos }}\right)$, is an increasing function of $\tau_{\text {eff }}$, depending on the parameters $t_{\text {int }}$ and $T$, that for small value of $t_{\text {int }}$ is enough linear for low lifetime. For measurements performed with $t_{\text {int }}=300 \mu \mathrm{s}$ and $T=$ $25 \mathrm{~ms}$ the function results are linear for lifetime smaller than $200 \mu \mathrm{s}$, with a sensitivity of $0.25^{\circ} \times \mu \mathrm{s}^{-1}$ (see the right side of Figure 10(a)). They conclude that if a shorter integration time is chosen, the sensitivity increases, and the linear range shifts toward the lower lifetime values.

\section{Conclusion}

In this paper we have examined all two optical techniques to measure the recombination lifetime in the semiconductor materials: the Transient Pump and Probe and the Infrared Lifetime Mapping. Both techniques are based on the Free Carriers Absorption and can be classified as Pump and Probe methods, since they make use of the common operation principle that uses a sub-band-gap radiation as probe to monitor the absorption variation in a sample induced by the generation of excess free carriers by means of a pump radiation.

In the TPP method the signal, where the interesting quantities are retrieved, is the transient evolution of the excess carrier density. This is generated by the pump pulse just after the turn off of the latter. Its main merit is the possibility to directly determine from the measurement a simultaneous evaluation of the bulk recombination lifetime and of the surface recombination velocity. Moreover, we have shown that little variations in the setup configuration give the method the flexibility to measure other electrical parameters of the sample. Furthermore, it allows for measuring very short lifetime, of the order of few nanosecond (depending on the pump pulse width).

The ILM, on the other hand, allows a very fast mapping of the actual recombination lifetime on the sample. In fact this method uses an infrared camera sensor to detect the free carriers absorption, so that the local information on the carrier density can be derived in a single acquisition without scanning the sample. Having additional knowledge about the carrier generation rate $G$, the effective carrier lifetime, $\tau_{\text {eff }}=\Delta n / G$, of the sample is calculated. In spite of being a steady-state method, based on the measurement of the frequency response of the semiconductor when excited by a modulated laser beam, it presents the disadvantages that the correct determination of the lifetime critically depends on the accuracy of the calibration procedure applied and on the accurate accounting of the lateral inhomogeneities in the optical properties of the sample. We have illustrated as this disadvantage is surmounted by the its dynamic version, which releases the measurements from the calibration, so that the methods results are to be perfectly complementary with respect to the TPP.

\section{Conflict of Interests}

The authors declare that there is no conflict of interests regarding the publication of this paper. 


\section{References}

[1] Z. G. Ling, P. K. Ajmera, M. Anselment, and L. F. Dimauro, "Lifetime measurements in semiconductors by infrared absorption due to pulsed optical excitation," Applied Physics Letters, vol. 51, no. 18, pp. 1445-1447, 1987.

[2] M. Bail, J. Kentsch, R. Brendel, and M. Schulz, "Lifetime mapping of $\mathrm{Si}$ wafers by an infrared camera [for solar cellproduction]," in Proceedings of the 28th Photovoltaic Specialists Conference, pp. 99-103, IEEE, Anchorage, Alaska, USA, 2000.

[3] S. Riepe, J. Isenberg, C. Ballif, S. Glunz, and W. Warta, "Carrier density and lifetime imaging of silicon wafers by infrared lock-in thermography," in Proceedings of the 17th European Photovoltaic Solar Energy Conference, pp. 1597-1599, 2001.

[4] N. Ashcroft and N. Mermin, Solid State Physics, Cengage Learning, Singapore, 1976.

[5] C. Kittel and P. McEuen, Introduction to Solid State Physics, Wiley, New York, NY, USA, 1996.

[6] J. Nelson, The Physics of Solar Cells, Imperial College Press, 2003.

[7] V. K. Khanna, "Physical understanding and technological control of carrier lifetimes in semiconductor materials and devices: a critique of conceptual development, state of the art and applications," Progress in Quantum Electronics, vol. 29, no. 2, pp. 59-163, 2005.

[8] P. Landsberg, Recombination in Semiconductors, Cambridge University Press, 1991.

[9] R. N. Hall, "Electron-hole recombination in germanium," Physical Review, vol. 87, no. 2, p. 387, 1952.

[10] W. Shockley and W. T. Read, "Statistics of the recombinations of holes and electrons," Physical Review, vol. 87, no. 5, pp. 835-842, 1952.

[11] V. K. Khanna, "Carrier lifetimes and recombination-generation mechanisms in semiconductor device physics," European Journal of Physics, vol. 25, no. 2, pp. 221-237, 2004.

[12] L. Pincherle, "Auger effect in semiconductors," Proceedings of the Physical Society B, vol. 68, no. 5, article 108, pp. 319-320, 1955.

[13] T. S. Moss, "Photoelectromagnetic and photoconductive effects in lead sulphide single crystals," Proceedings of the Physical Society B, vol. 66, no. 12, article 301, pp. 993-1002, 1953.

[14] J. A. Hornbeck and J. R. Haynes, "Trapping of minority carriers in silicon. I. P-type silicon," Physical Review, vol. 97, no. 2, pp. 311-321, 1955.

[15] P. Auger, "The compound photoelectric effect," Journal de Physique et le Radium, vol. 6, no. 6, article 205, 1925.

[16] P. T. Landsberg and T. S. Moss, "Recombination theory for indium antimonide," Proceedings of the Physical Society B, vol. 69, no. 6, article 310, pp. 661-669, 1956.

[17] P. T. Landsberg, "Lifetimes of excess carriers in InSb," Proceedings of the Physical Society B, vol. 70, no. 12, article 109, pp. 1175$1176,1957$.

[18] A. Beattie and P. Landsberg, "Auger effect in semiconductors," Proceedings of the Royal Society of London A: Mathematical and Physical Sciences, vol. 249, no. 1256, pp. 16-29, 1959.

[19] A. B. Grebene, "Comments on auger recombination in semiconductors," Journal of Applied Physics, vol. 39, no. 10, pp. 48664868, 1968.

[20] P. Lal, C. Rhys-Roberts, and P. Landsberg, Auger Recombination Into Traps, Ft. Belvoir Defense Technical Information Center, 1964.

[21] P. T. Landsberg, "Trap-Auger recombination in silicon of low carrier densities," Applied Physics Letters, vol. 50, no. 12, pp. 745747, 1987.
[22] J. Dziewior and W. Schmid, "Auger coefficients for highly doped and highly excited silicon," Applied Physics Letters, vol. 31, no. 5, pp. 346-348, 1977.

[23] D. J. Fitzgerald and A. S. Grove, "Surface recombination in semiconductors," Surface Science, vol. 9, no. 2, pp. 347-369, 1968.

[24] P. T. Landsberg, "Some general recombination statistics for semiconductor surfaces," IEEE Transactions on Electron Devices, vol. ED-29, no. 8, pp. 1284-1286, 1982.

[25] D. E. Aspnes, "Recombination at semiconductor surfaces and interfaces," Surface Science, vol. 132, no. 1-3, pp. 406-421, 1983.

[26] G. J. Rees, "Surface recombination velocity: a useful concept?" Solid State Electronics, vol. 28, no. 5, pp. 517-519, 1985.

[27] D. K. Schröder, "Carrier lifetimes in silicon," IEEE Transactions on Electron Devices, vol. 44, no. 1, pp. 160-170, 1997.

[28] H. B. Briggs, "Infra-red absorption in silicon," Physical Review, vol. 77, no. 5, pp. 727-728, 1950.

[29] H. Fan and M. Becker, "Infra-red optical properties of silicon and germanium," in Semi-Conducting Materials: Proceedings of a Conference Held at the University of Reading under the Auspices of the International Union of Pure and Applied Physics, in cooperation with the Royal Society, p. 132, Butterworths Scientific Publications, 1951.

[30] H. B. Briggs and R. C. Fletcher, "New infrared absorption bands in p-type germanium," Physical Review, vol. 87, no. 6, pp. 11301131, 1952.

[31] H. B. Briggs and R. C. Fletcher, "Absorption of infrared light by free carriers in germanium," Physical Review, vol. 91, no. 6, pp. 1342-1346, 1953.

[32] A. H. Kahn, "Theory of the infrared absorption of carriers in germanium and silicon," Physical Review, vol. 97, no. 6, pp.16471652, 1955.

[33] H. Y. Fan, W. Spitzer, and R. J. Collins, "Infrared absorption in n-type germanium," Physical Review, vol. 101, no. 2, pp. 566-572, 1956.

[34] W. Spitzer and H. Y. Fan, "Infrared absorption in $n$-type silicon," Physical Review, vol. 108, no. 2, pp. 268-271, 1957.

[35] S. Visvanathan, "Free carrier absorption due to polar modes in the III-V compound semiconductors," Physical Review, vol. 120, no. 2, pp. 376-378, 1960.

[36] W. P. Dumke, "Quantum theory of free carrier absorption," Physical Review, vol. 124, no. 6, pp. 1813-1817, 1961.

[37] H. Hara and Y. Nishi, "Free carrier absorption in p-type silicon," Journal of the Physical Society of Japan, vol. 21, no. 6, p. 1222, 1966.

[38] W. Runyan, Technology Semiconductor Silicon, 1966.

[39] D. Schroder, R. Thomas, and J. Swartz, "Free carrier absorption in silicon," IEEE Transactions on Electron Devices, vol. 25, pp. 254-261, 1978.

[40] J. I. Pankove, Optical Processes in Semi-Conductors, Dover Publications, 1971.

[41] P. Basu, Theory of Optical Processes in Semiconductors: Bulk and Microstructures, Oxford University Press, New York, NY, USA, 1997.

[42] B. Nag, Electron Transport in Compound Semiconductors, Springer, 1980.

[43] G. Chiarotti and U. M. Grassano, "The excited states of the F center investigated by means of modulated optical absorption," Il Nuovo Cimento B Series 10, vol. 46, no. 1, pp. 78-92, 1966.

[44] G. Chiarotti and U. M. Grassano, "Modulated F-center absorption in KCl," Physical Review Letters, vol. 16, no. 4, pp. 124-127, 1966. 
[45] W. B. Gauster and J. C. Bushnell, "Laser-induced infrared absorption in silicon," Journal of Applied Physics, vol. 41, no. 9, pp. 3850-3853, 1970.

[46] E. J. Conway, "Light-induced modulation of broad-band optical absorption in CdS," Journal of Applied Physics, vol. 41, no. 4, pp. 1689-1693, 1970.

[47] F. Sanii, R. J. Schwartz, R. F. Pierret, and W. M. Au, "Measurement of bulk and surface recombination by means of modulated free carrier absorption," in Proceedings of the 20th IEEE Photovoltaic Specialists Conference, pp. 575-580, September 1988.

[48] F. P. Giles, F. Sanii, R. J. Schwartz, and J. L. Gray, "Nondestructive contactless measurement of bulk lifetime and surface recombination using single pass infrared free carrier absorption," in Proceedings of the 22nd IEEE Photovoltaic Specialists Conference, pp. 223-228, October 1991.

[49] S. W. Glunz, A. B. Sproul, W. Warta, and W. Wettling, "Injectionlevel-dependent recombination velocities at the $\mathrm{Si}_{-} \mathrm{SiO}_{2}$ interface for various dopant concentrations," Journal of Applied Physics, vol. 75, no. 3, pp. 1611-1615, 1994.

[50] S. W. Glunz and W. Warta, "High-resolution lifetime mapping using modulated free-carrier absorption," Journal of Applied Physics, vol. 77, no. 7, pp. 3243-3247, 1995.

[51] Z. G. Ling and P. K. Ajmera, "Measurement of bulk lifetime and surface recombination velocity by infrared absorption due to pulsed optical excitation," Journal of Applied Physics, vol. 69, no. 1, pp. 519-521, 1991.

[52] G. S. Kousik, Z. G. Ling, and P. K. Ajmera, "Nondestructive technique to measure bulk lifetime and surface recombination velocities at the two surfaces by infrared absorption due to pulsed optical excitation," Journal of Applied Physics, vol. 72, no. 1, pp. 141-146, 1992.

[53] T. Otaredian, "Separate contactless measurement of the bulk lifetime and the surface recombination velocity by the harmonic optical generation of the excess carriers," Solid-State Electronics, vol. 36, no. 2, pp. 153-162, 1993.

[54] Z. G. Ling, P. K. Ajmera, and G. S. Kousik, "Simultaneous extraction of bulk lifetime and surface recombination velocities from free carrier absorption transients," Journal of Applied Physics, vol. 75, no. 5, pp. 2718-2720, 1994.

[55] K. L. Luke and L.-J. Cheng, "Analysis of the interaction of a laser pulse with a silicon wafer: determination of bulk lifetime and surface recombination velocity," Journal of Applied Physics, vol. 61, no. 6, pp. 2282-2293, 1987.

[56] J. Linnros, "Carrier lifetime measurements using free carrier absorption transients. I: principle and injection dependence," Journal of Applied Physics, vol. 84, no. 1, pp. 275-283, 1998.

[57] J. Linnros, "Carrier lifetime measurements using free carrier absorption transients. II. Lifetime mapping and effects of surface recombination," Journal of Applied Physics, vol. 84, no. 1, pp. 284-291, 1998.

[58] R. Bernini, A. Cutolo, A. Irace, P. Spirito, and L. Zeni, "Contactless characterization of the recombination process in silicon wafers: separation between bulk and surface contribution," Solid-State Electronics, vol. 39, no. 8, pp. 1165-1172, 1996.

[59] A. Irace, L. Sirleto, G. F. Vitale et al., "Transverse probe optical lifetime measurement as a tool for in-line characterization of the fabrication process of a silicon solar cell," Solid-State Electronics, vol. 43, no. 12, pp. 2235-2242, 1999.

[60] M. C. Schubert, S. Riepe, S. Bermejo, and W. Warta, "Determination of spatially resolved trapping parameters in silicon with injection dependent carrier density imaging," Journal of Applied Physics, vol. 99, no. 11, Article ID 114908, 2006.
[61] K. Ramspeck, S. Reissenweber, J. Schmidt, K. Bothe, and R. Brendel, "Dynamic carrier lifetime imaging of silicon wafers using an infrared-camera-based approach," Applied Physics Letters, vol. 93, no. 10, Article ID 102104, 2008.

[62] A. Buczkowski, Z. J. Radzimski, G. A. Rozgonyi, and F. Shimura, "Bulk and surface components of recombination lifetime based on a two-laser microwave reflection technique," Journal of Applied Physics, vol. 69, no. 9, pp. 6495-6499, 1991.

[63] L. Sirleto, A. Irace, G. F. Vitale, L. Zeni, and A. Cutolo, "Separation of bulk lifetime and surface recombination velocity by multiwavelength technique," Electronics Letters, vol. 38, no. 25, pp. 1742-1743, 2002.

[64] L. Sirleto, A. Irace, G. F. Vitale, L. Zeni, and A. Cutolo, "Separation of bulk lifetime and surface recombination velocity obtained by transverse optical probing and multi-wavelength technique," Optics and Lasers in Engineering, vol. 38, no. 6, pp. 461-472, 2002

[65] L. Sirleto, A. Irace, G. Vitale, L. Zeni, and A. Cutolo, "All-optical multiwavelength technique for the simultaneous measurement of bulk recombination lifetimes and front/rear surface recombination velocity in single crystal silicon samples," Journal of Applied Physics, vol. 93, no. 6, pp. 3407-3413, 2003.

[66] A. Irace, L. Sirleto, P. Spirito et al., "Optical characterization of the recombination process in silicon wafers, epilayers and devices," Optics and Lasers in Engineering, vol. 39, no. 2, pp. 219232, 2003.

[67] A. Irace, F. Sorrentino, and G. F. Vitale, "Multi-wavelength transverse probe lifetime measurement for the characterization of recombination lifetime in thin mc-Si samples for photovoltaic industry use," Solar Energy Materials and Solar Cells, vol. 84, no. 1-4, pp. 83-92, 2004.

[68] A. Irace, F. Sorrentino, and G. F. Vitale, "Multi-wavelength all optical measurement for the characterization of recombination process in thin mc-Si samples," Solar Energy, vol. 78, no. 2, pp. 251-256, 2005.

[69] E. Gaubas and J. Vanhellemont, "A simple technique for the separation of bulk and surface recombination parameters in silicon," Journal of Applied Physics, vol. 80, no. 11, pp. 6293-6297, 1996.

[70] E. Gaubas, J. Vaitkus, E. Simoen, C. Claeys, and J. Vanhellemont, "Excess carrier cross-sectional profiling technique for determination of the surface recombination velocity," Materials Science in Semiconductor Processing, vol. 4, no. 1-3, pp. 125-131, 2001.

[71] M. De Laurentis, A. Irace, and G. Breglio, "Accurate modelling of the pump-probe spatial interaction in an all-optical recombination lifetime measurement setup," in Proceedings of the 1st Mediterranean Photonics Conference, p. 40, Ischia, Italy, 2008.

[72] M. De Laurentis, A. Irace, and G. Breglio, "Determination of the surface electrical activity in silicon wafers with a laser pumpprobe measurement," in Proceedings of the 6th International Conference on Photo-Excited Processes and Applications, p. 121, 2008.

[73] A. G. Aberle, J. Schmidt, and R. Brendel, "On the data analysis of light-biased photoconductance decay measurements," Journal of Applied Physics, vol. 79, no. 3, pp. 1491-1496, 1996.

[74] J. Schmidt and A. G. Aberle, "Accurate method for the determination of bulk minority-carrier lifetimes of mono- and multicrystalline silicon wafers," Journal of Applied Physics, vol. 81, no. 9, pp. 6186-6199, 1997.

[75] F. M. Schuurmans, A. Schönecker, A. R. Burgers, and W. C. Sinke, "Simplified evaluation method for light-biased effective 
lifetime measurements," Applied Physics Letters, vol. 71, no. 13, pp. 1795-1797, 1997.

[76] J. Schmidt, "Measurement of differential and actual recombination parameters on crystalline silicon wafers," IEEE Transactions on Electron Devices, vol. 46, no. 10, pp. 2018-2025, 1999.

[77] O. Breitenstein and M. Langenkamp, Lock-in Thermography: Basics and Use for Functional Diagnostics of Electronic Components, Springer, 2003.

[78] W. Warta, "Advanced defect and impurity diagnostics in silicon based on carrier lifetime measurements," Physica Status Solidi (A) Applications and Materials Science, vol. 203, no. 4, pp. 732746, 2006.

[79] M. C. Schubert, J. Isenberg, and W. Warta, "Spatially resolved lifetime imaging of silicon wafers by measurement of infrared emission," Journal of Applied Physics, vol. 94, no. 6, pp. 41394143, 2003.

[80] J. Isenberg, S. Riepe, S. W. Glunz, and W. Warta, "Carrier density imaging (CDI): a spatially resolved lifetime measurement suitable for in-line process-control," in Proceedings of the 29th IEEE Photovoltaic Specialists Conference, pp. 266-269, May 2002.

[81] J. Isenberg, S. Riepe, S. W. Glunz, and W. Warta, "Imaging method for laterally resolved measurement of minority carrier densities and lifetimes: measurement principle and first applications," Journal of Applied Physics, vol. 93, no. 7, pp. 4268-4275, 2003.

[82] P. Pohl and R. Brendel, "Temperature dependent infrared camera lifetime mapping (ILM)," in Proceedings of the 19th European Photovoltaic Solar Energy Conference, pp. 46-49, Paris, France, 2004. 

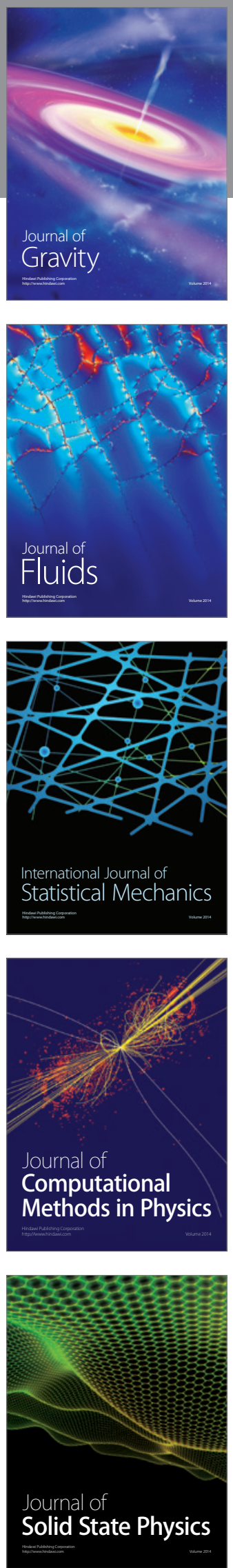

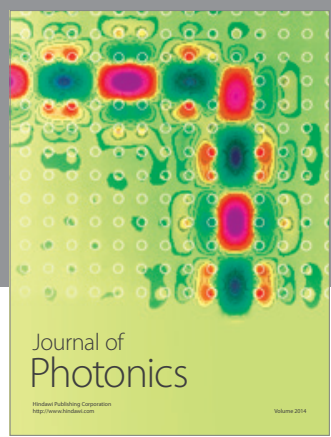

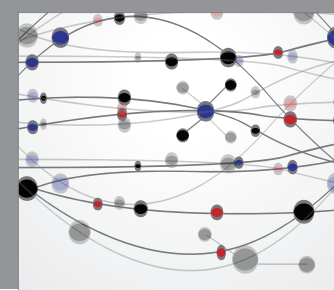

The Scientific World Journal



Soft Matter
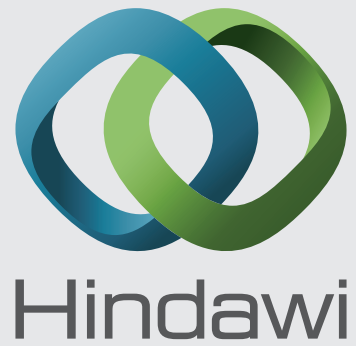

Submit your manuscripts at

http://www.hindawi.com
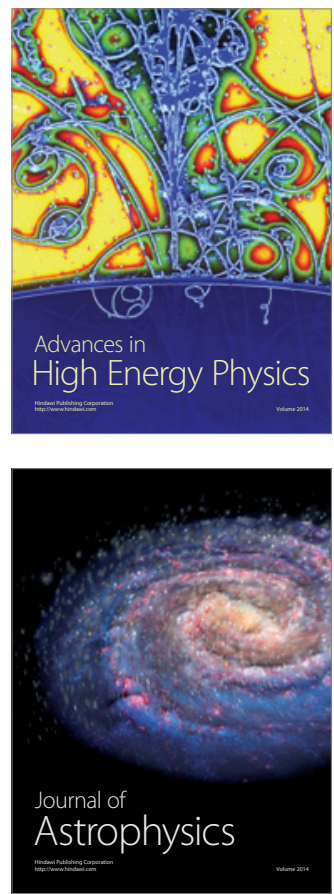
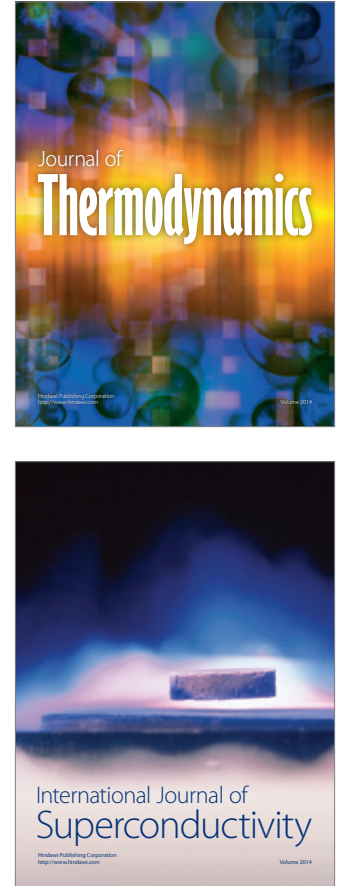
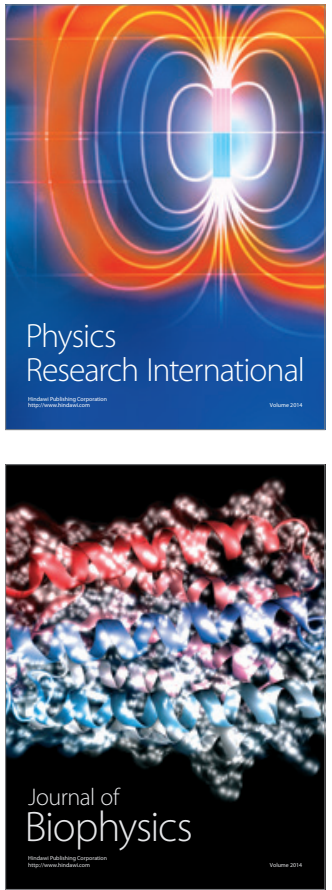
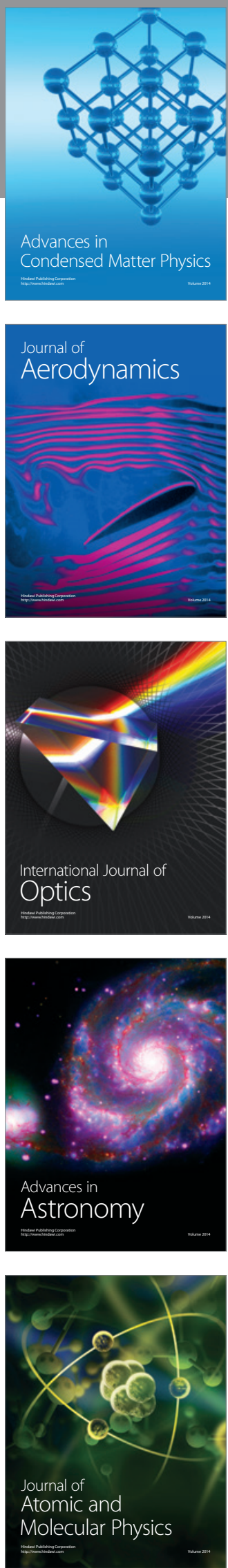